\title{
LOCALIZATION AND CONJECTURES FROM STRING DUALITY
}

\author{
KEFENG LIU
}

\begin{abstract}
We describe the applications of localization methods, in particular the functorial localization formula, in the proofs of several conjectures from string theory. Functorial localization formula pushes the computations on complicated moduli spaces to simple moduli spaces. It is a key technique in the proof of the general mirror formulas, the proof of the Hori-Vafa formulas for explicit expressions of basic hypergeometric series of homogeneous manifolds, the proof of the Mariño-Vafa formula, its generalizations to two partition analogue. We will also discuss our development of the mathematical theory of topological vertex and simple localization proofs of the ELSV formula and Witten conjecture.
\end{abstract}

\section{INTRODUCTION}

According to string theorists, String Theory, as the most promising candidate for the grand unification of all fundamental forces in the nature, should be the final theory of the world, and should be unique. But now there are five different looking string theories. As argued by physicists, these theories should be equivalent, in a way dual to each other. On the other hand all previous theories like the Yang-Mills and the Chern-Simons theory should be parts of string theory. In particular their partition functions should be equal or equivalent to each other in the sense that they are equal after certain transformation. To compute partition functions, physicists use localization technique, a modern version of residue theorem, on infinite dimensional spaces. More precisely they apply localization formally to path integrals which is not well-defined yet in mathematics. In many cases such computations reduce the path integrals to certain integrals of various Chern classes on various finite dimensional moduli spaces, such as the moduli spaces of stable maps and the moduli spaces of vector bundles. The identifications of these partition functions among different theories have produced many surprisingly beautiful mathematical formulas like the famous mirror formula [7, as well as the Mariño-Vafa formula [46].

The mathematical proofs of these conjectural formulas from the string duality also depend on localization techniques on these various finite dimensional moduli spaces. The purpose of this note is to discuss our works on the subject. I will briefly discuss the proof of the mirror conjecture and its generalizations, the proof of the Hori-Vafa formula, the proof of the Marinõ-Vafa formula and its generalizations, the related topological vertex theory [1] [28], and simple localization proofs of the ELSV formula and the Witten conjecture [22]. More precisely we will use localization formulas in various form to compute the integrals of Chern classes on moduli spaces, and to prove those conjectures from string duality. For the proofs of these conjectures such as the mirror formula, the Mariño-Vafa formula and the theory of topological vertex, we note that many aspects of mathematics are involved, such as the Chern-Simons knot invariants, combinatorics of symmetric groups, representations

Proceedings of the 23rd International Conference of Differential Geometric Methods in Theoretical Physics Tianjin, 20 - 26 August 2005. The author is supported by the NSF and NSFC. 
of Kac-Moody algebras, Calabi-Yau manifolds, geometry and topology of moduli space of stable maps, etc. The spirit of our results is the duality among various string theories. In particular the duality between IIA and IIB string theory gives the mirror formulas, the duality between gauge theory, Chern-Simons theory and the Calabi-Yau geometry in string theory leads the Mariño-Vafa conjecture and the theory of topological vertex.

Localization techniques have been very successful in proving many conjectures from physics, see my ICM 2002 lecture [43] for more examples. One of our major tools in the proofs of these conjectures is the functorial localization formula which is a variation of the classical localization formula, it transfers computations on complicated spaces to simple spaces, and connects computations of mathematicians and physicists.

In this note we will discuss the following results:

1. The proof of the mirror formulas and its generalizations which we call the mirror principle. The mirror principle implies all of the conjectural mirror formulas of counting rational curves for toric manifolds and their Calabi-Yau submanifolds from string theory. In this case we apply the functorial localization formula to the map from the nonlinear moduli space to the linearized moduli space. This transfers the computations of integrals on complicated moduli space of stable maps to computations on rather simple spaces like projective spaces. From this the proof of the mirror formula and its generalizations become conceptually clean and simple.

In fact the functorial localization formula was first found and used in Lian-LiuYau's proof of the mirror conjecture.

2. The proof of the Hori-Vafa conjecture and its generalizations for Grassmannian and flag manifolds. This conjecture predicts an explicit formula for the basic hypergeometric series of a homogeneous manifold in terms of the basic series of a simpler manifold such as the product of projective spaces. In this case we use the functorial localization formula twice to transfer the computations on the complicated moduli spaces of stable maps to the computations on quot-schemes. The first is a map from moduli space of stable maps to product of projective spaces, and another one is a map from the quot-scheme into the same product of projective spaces. A key observation we had is that these two maps have the same image.

This approach was first sketched in [33], the details for Grassmannians were carried out in [30] and [4]. The most general case of flag manifolds was carried out in [37] and [5].

3. The proof of the Mariño-Vafa conjecture on Hodge integrals in [40]. This conjecture gives a closed formula for the generating series of a class of triple Hodge integrals for all genera and any number of marked points in terms of the ChernSimons knot invariant of the unknot. This formula was conjectured by M. Mariño and C. Vafa in [46] based on the duality between large $N$ Chern-Simons theory and string theory. Many Hodge integral identities, including the ELSV formula for Hurwitz numbers [10] and the $\lambda_{g}$ conjecture [12], can be obtained by taking various limits of the Mariño-Vafa formula [41. The Mariño-Vafa formula was first proved by applying the functorial localization formula to the branch morphism from the moduli space of relative stable maps to a projective space. 
4. The proof of the generalization of the Mariño-Vafa formula to two partitions cases, and the theory of topological vertex. The mathematical theory of topological vertex was motivated by the physical theory as first developed by the Vafa group [1], who has been working on string duality for the past several years. Topological vertex theory is a high point of their work starting from their geometric engineering theory and Witten's conjecture that Chern-Simons theory is a string theory [51]. While the Marinõ-Vafa formula gives a close formula for the generating series of triple Hodge integrals on the moduli spaces of all genera and any number marked points, topological vertex [28] gives the most effective ways to compute the Gromov-Witten invariants of any open toric Calabi-Yau manifolds. Recently Pan Peng was able to use our results on topological vertex to give a complete proof of the Gopakumar-Vafa integrality conjecture for any open toric Calabi-Yau manifolds [48]. Kim also used our technique to derive new effective recursion formulas for Hodge integrals on the moduli spaces of stable curves [20].

5. We describe a very simple proof of the ELSV formula [10] following our proof of the Mariño-Vafa formula, by using the cut-and-join equation from localization and combinatorics. The proof of the ELSV formula is particularly easy by using functorial localization, it is reduced to the fact that the push-forward in equivariant cohomology of a constant between two equal dimensional varieties is still constant. We will also show how to directly derive the ELSV formula from the Mariño-Vafa formula by taking a direct limit.

6. By using functorial localization formula we have the simple proofs of the Witten conjecture [22]. Our simple proof of the Witten conjecture in [21] is to study the asymptotic expansion of the simple cut-and-join equation for one Hodge integrals which is derived from functorial localization. This immediately gives a recursion formula which implies both the Virasoro constraints and the KdV relation satisfied by the generating series of the $\psi$ integrals.

I will start with brief discussions about the proofs of the mirror conjecture and the HoriVafa formula for Grassmannians, then I will go to the proofs of the Marinõ-Vafa conjecture and its generalizations to two partitions and the topological vertex theory. After that we discuss the simple proofs of the ELSV formula and the Witten conjecture. This note is basically a detailed account of my plenary lecture at the International Conference of Differential Geometry Method in Theoretical Physics held in August 2005. It is an much more expanded version of a previous survey I wrote for the 2004 International Complex Geometry Conference held in the Eastern Normal University of China. I would like to thank the organizers of the conferences, especially Professor Chunming Bai, Professor Shengli Tan, Professor Weiping Zhang and Professor Zhijie Chen for their hospitality during my visits. I would also like to thank my collaborators for the past 10 years, Bong Lian, Shing-Tung Yau, Chien-Hao Liu, Melissa C.-C. Liu, Jian Zhou, Jun Li, Yon Seo Kim for the wonderful experience in solving these conjectures and to develop the theory together.

\section{LOCALIZATION}

In this section we will explain the Functorial Localization Formula. We start with a review of the Atiyah-Bott localization formula. Recall that the definition of equivariant 
cohomology group for a manifold $X$ with a torus $T$ action:

$$
H_{T}^{*}(X)=H^{*}\left(X \times_{T} E T\right)
$$

where ET is the universal bundle of $T$, we will use $\mathbb{R}$ or $\mathbb{Q}$ as coefficients through this note.

Example. We know $E S^{1}=S^{\infty}$. If $S^{1}$ acts on $\mathbf{P}^{n}$ by

$$
\lambda \cdot\left[Z_{0}, \ldots, Z_{n}\right]=\left[\lambda^{w_{0}} Z_{0}, \ldots, \lambda^{w_{n}} Z_{n}\right]
$$

with $w_{0}, \cdots, w_{n}$ as weights, then

$$
H_{S^{1}}^{*}\left(\mathbf{P}^{n} ; \mathbb{Q}\right) \cong \mathbb{Q}[H, u] /\left\langle\left(H-w_{0} u\right) \cdots\left(H-w_{n} u\right)\right\rangle
$$

where $u$ is the generator of $H^{*}\left(B S^{1}, \mathbb{Q}\right)$. We have the following important Atiyah-Bott Localization Formula:

Theorem 2.1. For $\omega \in H_{T}^{*}(X)$ an equivariant cohomology class, we have

$$
\omega=\sum_{E} i_{E *}\left(\frac{i_{E}^{*} \omega}{e_{T}(E / X)}\right)
$$

where $E$ runs over all connected components of $T$ fixed points set, $i_{E}$ denotes the inclusion map, $i_{E}^{*} i_{E *}$ denote the pull-back and push-forward in equivariant cohomology.

This formula is very effective in the computations of integrals on manifolds with torus $T$ symmetry. The idea of localization is fundamental in many subjects of geometry. In fact Atiyah and Witten proposed to formally apply this localization formula to loop spaces and the natural $S^{1}$-action, from which one gets the Atiyah-Singer index formula. In fact the Chern characters can be interpreted as equivariant forms on loop space, and the $\hat{A}$-class is the inverse of the equivariant Euler class of the normal bundle of $X$ in its loop space $L X$ :

$$
e_{T}(X / L X)^{-1} \sim \hat{A}(X)
$$

which follows from the normalized infinite product formula

$$
\left(\prod_{n \neq 0}(x+n)\right)^{-1} \sim \frac{x}{\sin x}
$$

I observed in 44] that the normalized product

$$
\prod_{m, n}(x+m+n \tau)=2 q^{\frac{1}{8}} \sin (\pi x) \cdot \prod_{j=1}^{\infty}\left(1-q^{j}\right)\left(1-e^{2 \pi i x} q^{j}\right)\left(1-e^{-2 \pi i x} q^{j}\right),
$$

where $q=e^{2 \pi i \tau}$, also has deep geometric meaning. This formula is the Eisenstein formula. It can be viewed as a double loop space analogue of the Atiyah-Witten observation. This formula gives the basic Jacobi $\theta$-function. As observed by in [44], formally this gives the $\hat{A}$-class of the loop space, and the Witten genus which is defined to be the index of the Dirac operator on the loop space:

$$
e_{T}(X / L L X) \sim \hat{W}(X)
$$

where $L L X$ is the double loop space, the space of maps from $S^{1} \times S^{1}$ into $X . \hat{W}(X)$ is the Witten class. See [44] for more detail. 
The variation of the localization formula we will use in various situations is the following Functorial Localization Formula

Theorem 2.2. Let $X$ and $Y$ be two manifolds with torus action. Let $f: X \rightarrow Y$ be an equivariant map. Given $F \subset Y$ a fixed component, let $E \subset f^{-1}(F)$ be those fixed components inside $f^{-1}(F)$. Let $f_{0}=\left.f\right|_{E}$, then for $\omega \in H_{T}^{*}(X)$ an equivariant cohomology class, we have the following identity on $F$ :

$$
f_{0 *}\left[\frac{i_{E}^{*} \omega}{e_{T}(E / X)}\right]=\frac{i_{F}^{*}\left(f_{*} \omega\right)}{e_{T}(F / Y)} .
$$

This formula will be applied to various settings to prove various conjectures from physics. It first appeared in [31. In many cases we will use a virtual version of this formula. It is used to push computations on complicated moduli spaces to simpler moduli spaces. A $K$-theory version of the functorial localization formula also holds [32, interesting applications are expected.

Remark. Consider the diagram:

$$
\begin{array}{ccc}
H_{T}^{*}(X) & \stackrel{f_{*}}{\longrightarrow} & H_{T}^{*}(Y) \\
\downarrow i_{E}{ }^{*} & & \downarrow i_{F}{ }^{*} \\
H_{T}^{*}(E) & \stackrel{f_{0 *}}{\longrightarrow} & H_{T}^{*}(F) .
\end{array}
$$

The functorial localization formula is like Riemann-Roch with the inverted equivariant Euler classes of the normal bundle as "weights", in a way similar to the Todd class for the Riemann-Roch formula. In fact if we formally apply this formula to the map between the loop spaces of $X$ and $Y$, equivariant with respect to the rotation of the circle, we do formally get the differentiable Riemann-Roch formula. We believe this can be done rigorously by following Bismut's proof of the index formula which made rigorous of the above argument of Atiyah-Witten.

\section{Mirror PRINCIPLE}

There have been many discussions of mirror principle in the literature. Here we only give a brief account of the main ideas of the setup and proof of the mirror principle. We will use two most interesting examples to illustrate the algorithm. These two examples give proofs of the mirror formulas for toric manifolds as conjectured by string theorists.

The goal of mirror principle is to compute the characteristic numbers on moduli spaces of stable maps in terms of certain hypergeometric type series. This was motivated by mirror symmetry in string theory. The most interesting case is the counting of the numbers of curves which corresponds to the computations of Euler numbers. More generally we would like to compute the characteristic numbers and classes induced from the general Hirzebruch multiplicative classes such as the total Chern classes. The computations of integrals on moduli spaces of those classes pulled back through evaluation maps at the marked points and the general Gromov-Witten invariants can also be considered as part of mirror principle. Our hope is to develop a "black-box" method which makes easy the computations of the characteristic numbers and the Gromov-Witten invariants.

The general set-up of mirror principle is as follows. Let $X$ be a projective manifold, $\overline{\mathcal{M}}_{g, k}(d, X)$ be the moduli space of stable maps of genus $g$ and degree $d$ with $k$ marked points into $X$, modulo the obvious equivalence. The points in $\overline{\mathcal{M}}_{g, k}(d, X)$ are triples 
$\left(f ; C ; x_{1}, \cdots, x_{k}\right)$ where $f: C \rightarrow X$ is a degree $d$ holomorphic map and $x_{1}, \cdots, x_{k}$ are $k$ distinct smooth points on the genus $g$ curve $C$. The homology class $f_{*}([C])=d \in$ $H_{2}(X, \mathbb{Z})$ is identified as integral index $d=\left(d_{1}, \cdots, d_{n}\right)$ by choosing a basis of $H_{2}(X, \mathbb{Z})$, dual to the Kähler classes.

In general the moduli space may be very singular, and may even have different dimension for different components. To define integrals on such singular spaces, we need the virtual fundamental cycle of Li-Tian [27], and also Behrend-Fantechi [6] which we denote by $\left[\overline{\mathcal{M}}_{g, k}(d, X)\right]^{v}$. This is a homology class of the expected dimension

$$
2\left(c_{1}(T X)[d]+\left(\operatorname{dim}_{\mathbb{C}} X-3\right)(1-g)+k\right)
$$

on $\overline{\mathcal{M}}_{g, k}(d, X)$.

Let us consider the case $k=0$ first. Note that the expected dimension of the virtual fundamental cycle is 0 if $X$ is a Calabi-Yau 3-fold. This is the most interesting case for string theory.

The starting data of mirror principle are as follows. Let $V$ be a concavex bundle on $X$ which we defined as the direct sum of a positive and a negative bundle on $X$. Then $V$ induces a sequence of vector bundles $V_{d}^{g}$ on $\overline{\mathcal{M}}_{g, 0}(d, X)$ whose fiber at $\left(f ; C ; x_{1}, \cdots, x_{k}\right)$ is given by $H^{0}\left(C, f^{*} V\right) \oplus H^{1}\left(C, f^{*} V\right)$. Let $b$ be a multiplicative characteristic class. So far for all applications in string theory, $b$ is the Euler class.

The problem of mirror principle is to compute

$$
K_{d}^{g}=\int_{\left[\overline{\mathcal{M}}_{g, 0}(d, X)\right]^{v}} b\left(V_{d}^{g}\right) .
$$

More precisely we want to compute the generating series

$$
F(T, \lambda)=\sum_{d, g} K_{d}^{g} \lambda^{g} e^{d \cdot T}
$$

in terms of certain hypergeometric type series. Here $\lambda, T=\left(T_{1}, \cdots, T_{n}\right)$ are formal variables.

The most famous formula in the subject is the Candelas formula as conjectured by $\mathrm{P}$. Candelas, X. de la Ossa, P. Green, and L. Parkes [7]. This formula changed the history of the subject. More precisely, Candelas formula considers the genus 0 curves, that is, we want to compute the so-called $A$-model potential of a Calabi-Yau 3-fold $M$ given by

$$
\mathcal{F}_{0}(T)=\sum_{d \in H_{2}(M ; \mathbb{Z})} K_{d}^{0} e^{d \cdot T},
$$

where $T=\left(T_{1}, \ldots, T_{n}\right)$ are considered as the coordinates of the Kahler moduli of $M$, and $K_{d}^{0}$ is the genus zero, degree $d$ invariant of $M$ which gives the numbers of rational curves of all degree through the multiple cover formula [31]. The famous mirror conjecture asserts that there exists a mirror Calabi-Yau 3 -fold $M^{\prime}$ with $B$-model potential $\mathcal{G}(T)$, which can be computed by period integrals, such that

$$
\mathcal{F}(T)=\mathcal{G}(t)
$$

where $t$ accounts for coordinates of complex moduli of $M^{\prime}$. The map $t \mapsto T$ is called the mirror map. In the toric case, the period integrals are explicit solutions to the GKZsystem, that is the Gelfand-Kapranov-Zelevinsky hypergeometric series. While the Aseries are usually very difficult to compute, the B-series are very easy to get. This is 
the magic of the mirror formula. We will discuss the proof of the mirror principle which includes the proof of the mirror formula.

The key ingredients for the proof of the mirror principle consists of

(1) Linear and non-linear moduli spaces;

(2) Euler data and hypergeometric (HG) Euler data.

More precisely, the non-linear moduli is the moduli space $M_{d}^{g}(X)$ which is the stable map moduli of degree $(1, d)$ and genus $g$ into $\mathbf{P}^{1} \times X$. A point in $M_{d}^{g}(X)$ consists of a pair $(f, C): f: C \rightarrow \mathbf{P}^{1} \times X$ with $C$ a genus $g$ (nodal) curve, modulo obvious equivalence. The linearized moduli $W_{d}$ for toric $X$ were first introduced by Witten and used by Aspinwall-Morrison to do approximating computations.

Example. Consider the projective space $\mathbf{P}^{n}$ with homogeneous coordinate $\left[z_{0}, \cdots, z_{n}\right]$. Then the linearized moduli $W_{d}$ is defined as projective space with coordinates

$$
\left[f_{0}\left(w_{0}, w_{1}\right), \cdots, f_{n}\left(w_{0}, w_{1}\right)\right]
$$

where $f_{j}\left(w_{0}, w_{1}\right)$ 's are homogeneous polynomials of degree $d$.

This is the simplest compactification of the moduli spaces of degree $d$ maps from $\mathbf{P}^{1}$ into $\mathbf{P}^{n}$. The following lemma is important. See [34] for its proof. The $g=0$ case was given in [13] and in [31].

Lemma 3.1. There exists an explicit equivariant collapsing map

$$
\varphi: M_{d}^{g}\left(\mathbf{P}^{n}\right) \longrightarrow W_{d}
$$

For general projective manifold $X$, the nonlinear moduli $M_{d}^{g}(X)$ can be embedded into $M_{d}^{g}\left(\mathbf{P}^{n}\right)$. The nonlinear moduli $M_{d}^{g}(X)$ is very "singular" and complicated, but the linear moduli $W_{d}$ is smooth and simple. The embedding induces a map of $M_{d}^{g}(X)$ to $W_{d}$. Functorial localization formula pushed the computations onto $W_{d}$. Usually mathematical computations should be done on the moduli of stable maps, while physicists tried to use the linearized moduli to approximate the computations. So functorial localization formula connects the computations of mathematicians and physicists. In some sense the mirror symmetry formula is more or less the comparison of computations on nonlinear and linearized moduli.

Mirror principle has been proved to hold for balloon manifolds. A projective manifold $X$ is called balloon manifold if it admits a torus action with isolated fixed points, and if the following conditions hold. Let

$$
H=\left(H_{1}, \cdots, H_{k}\right)
$$

be a basis of equivariant Kahler classes such that

(1) the restrictions $H(p) \neq H(q)$ for any two fixed points $p \neq q$;

(2) the tangent bundle $T_{p} X$ has linearly independent weights for any fixed point $p$.

This notion was introduced by Goresky-Kottwitz-MacPherson.

Theorem 3.2. Mirror principle holds for balloon manifolds and for any concavex bundles.

\section{Remarks.}

1. All toric manifolds are balloon manifolds. For $g=0$ we can identify the hypergeometric series explicitly. Higher genus cases need more work to identify such series. 
2. For toric manifolds and $g=0$, mirror principle implies all of the mirror conjectural formulas from string theory.

3. For Grassmannian manifolds, the explicit mirror formula is given by the Hori-Vafa formula to be discussed in Section 4 .

4. The case of direct sum of positive line bundles on $\mathbf{P}^{n}$, including the Candelas formula. For proofs of this formula from two different points of view, see [31] and [13, 49, 3]; for a comparison of the two points of view, see [9].

Now we briefly discuss the proof of the mirror principle. The main idea is to apply the functorial localization formula to $\varphi$, the collapsing map and the pull-back class $\omega=$ $\pi^{*} b\left(V_{d}^{g}\right)$, where $\pi: M_{d}^{g}(X) \rightarrow \overline{\mathcal{M}}_{g, 0}(d, X)$ is the natural projection.

Such classes satisfy certain induction property. To be precise we introduce the notion of Euler Data, which naturally appears on the right hand side of the functorial localization formula, $Q_{d}=\varphi_{!}\left(\pi^{*} b\left(V_{d}^{g}\right)\right)$ which is a sequence of polynomials in equivariant cohomology rings of the linearized moduli spaces with simple quadratic relations. We also considered their restrictions to $X$.

From functorial localization formula we prove that, by knowing the Euler data $Q_{d}$ we can determine the $K_{d}^{g}$. On the other hand, there is another much simpler Euler data, the $H G$ Euler data $P_{d}$, which coincides with $Q_{d}$ on the "generic" part of the nonlinear moduli. We prove that the quadratic relations and the coincidence on generic part determine the Euler data uniquely up to certain degree. We also know that $Q_{d}$ always have the right degree for $g=0$. We then use mirror transformation to reduce the degrees of the HG Euler data $P_{d}$. From these we deduced the mirror principle.

\section{Remarks.}

1. Both the denominator and the numerator in the HG series, the generating series of the HG Euler data, are equivariant Euler classes. Especially the denominator is exactly from the localization formula. This is easily seen from the functorial localization formula.

2. The quadratic relation of Euler data, which naturally comes from gluing and functorial localization on the A-model side, is closely related to special geometry, and is similar to the Bershadsky-Cecotti-Ooguri-Vafa's holomorphic anomaly equation on the B-model side. Such relation can determine the polynomial Euler data up to certain degree.

It is an interesting task to use special geometry to understand the mirror principle computations, especially the mirror transformation as a coordinate change.

3. The Mariño-Vafa formula to be discussed later is needed to determine the hypergeometric Euler data for higher genus computations in mirror principle. The Mariño-Vafa formula comes from the duality between Chern-Simons theory and Gromov-Witten theory. This duality and the matrix model for Chern-Simons theory indicate that mirror principle may have matrix model description.

Let us use two examples to illustrate the algorithm of mirror principle.

Example. Consider the Calabi-Yau quintic in $\mathbf{P}^{4}$. In this case

$$
P_{d}=\prod_{m=0}^{5 d}(5 \kappa-m \alpha)
$$


with $\alpha$ can be considered as the weight of the $S^{1}$ action on $\mathbf{P}^{1}$, and $\kappa$ denotes the generator of the equivariant cohomology ring of $W_{d}$.

The starting data of the mirror principle in this case is $V=\mathcal{O}(5)$ on $X=\mathbf{P}^{4}$. The hypergeometric series, after taking $\alpha=-1$, is given by

$$
H G[B](t)=e^{H t} \sum_{d=0}^{\infty} \frac{\prod_{m=0}^{5 d}(5 H+m)}{\prod_{m=1}^{d}(H+m)^{5}} e^{d t},
$$

where $H$ is the hyperplane class on $\mathbf{P}^{4}$ and $t$ is a formal parameter.

We introduce the series

$$
\mathcal{F}(T)=\frac{5}{6} T^{3}+\sum_{d>0} K_{d}^{0} e^{d T}
$$

The algorithm is as follows. Take the expansion in $H$ :

$$
H G[B](t)=H\left\{f_{0}(t)+f_{1}(t) H+f_{2}(t) H^{2}+f_{3}(t) H^{3}\right\},
$$

from which we have the famous Candelas Formula: With $T=f_{1} / f_{0}$,

$$
\mathcal{F}(T)=\frac{5}{2}\left(\frac{f_{1}}{f_{0}} \frac{f_{2}}{f_{0}}-\frac{f_{3}}{f_{0}}\right) .
$$

Example. Let $X$ be a toric manifold and $g=0$. Let $D_{1}, . ., D_{N}$ be the $T$-invariant divisors in $X$. The starting data consist of $V=\oplus_{i} L_{i}$ with $c_{1}\left(L_{i}\right) \geq 0$ and $c_{1}(X)=c_{1}(V)$. Let us take $b(V)=e(V)$ the Euler class. We want to compute the A-series

$$
A(T)=\sum K_{d}^{0} e^{d \cdot T} .
$$

The HG Euler series which is the generating series of the HG Euler data can be easily written down as

$$
B(t)=e^{-H \cdot t} \sum_{d} \prod_{i} \prod_{k=0}^{\left\langle c_{1}\left(L_{i}\right), d\right\rangle}\left(c_{1}\left(L_{i}\right)-k\right) \frac{\prod_{\left\langle D_{a}, d\right\rangle<0} \prod_{k=0}^{-\left\langle D_{a}, d\right\rangle-1}\left(D_{a}+k\right)}{\prod_{\left\langle D_{a}, d\right\rangle \geq 0} \prod_{k=1}^{\left\langle D_{a}, d\right\rangle}\left(D_{a}-k\right)} e^{d \cdot t} .
$$

Then mirror principle implies that there are explicitly computable functions $f(t), g(t)$, which define the mirror map, such that

$$
\int_{X}\left(e^{f} B(t)-e^{-H \cdot T} e(V)\right)=2 A(T)-\sum T_{i} \frac{\partial A(T)}{\partial T_{i}}
$$

where $T=t+g(t)$. From this equation we can easily solve for $A(T)$.

In general we want to compute:

$$
K_{d, k}^{g}=\int_{\left[\mathcal{M}_{g, k}(d, X)\right]^{v}} \prod_{j=1}^{k} e v_{j}^{*} \omega_{j} \cdot b\left(V_{d}^{g}\right)
$$

where $\omega_{j} \in H^{*}(X)$ and $e v_{j}$ denotes the evaluation map at the $j$-th marked point. We form a generating series with $t, \lambda$ and $\nu$ formal variables,

$$
F(t, \lambda, \nu)=\sum_{d, g, k} K_{d, k}^{g} e^{d t} \lambda^{2 g} \nu^{k} .
$$

The ultimate mirror principle we want to prove is to compute this series in terms of certain explicit HG series. It is easy to show that those classes in the integrand can still 
be combined to induce Euler data. Actually the Euler data really encode the geometric structure of the stable map moduli.

We only use one example to illustrate the higher genus mirror principle.

Example. Consider open toric Calabi-Yau manifold, say $\mathcal{O}(-3) \rightarrow \mathbf{P}^{2}$. Here $V=\mathcal{O}(-3)$. Let

$$
Q_{d}=\sum_{g \geq 0} \varphi_{!}\left(\pi^{*} e_{T}\left(V_{d}^{g}\right)\right) \lambda^{2 g} .
$$

Then it can be shown that the corresponding HG Euler data is given explicitly by

$$
P_{d} J(\kappa, \alpha, \lambda) J(\kappa-d \alpha,-\alpha, \lambda),
$$

where $P_{d}$ is exactly the genus 0 HG Euler data and $J$ is generating series of Hodge integrals with summation over all genera. $J$ may be considered as the degree 0 Euler data. In fact we may say that the computations of Euler data include computations of all Gromov-Witten invariants, and even more. Zhou has obtained some closed formulas. We have proved that the mirror principle holds in such general setting. The remaining task is to determine the explicit HG Euler data. But the recently developed topological vertex theory has given complete closed formulas in terms of the Chern-Simons invariants. See the discussion in Section 7 for details.

Finally we mention some recent works. First we have constructed refined linearized moduli space for higher genus, the $A$-twisted moduli stack $\mathcal{A M}_{g}(X)$ of genus $g$ curves associated to a smooth toric variety $X$, induced from the gauged linear sigma model studied by Witten.

This new moduli space is constructed as follows. A morphism from a curve of genus $g$ into $X$ corresponds to an equivalence class of triples $\left(L_{\rho}, u_{\rho}, c_{m}\right)_{\rho, m}$, where each $L_{\rho}$ is a line bundle pulled back from $X, u_{\rho}$ is a section of $L_{\rho}$ satisfying a non-degeneracy condition, and the collection $\left\{c_{m}\right\}_{m}$ gives conditions to compare the sections $u_{\rho}$ in different line bundles $L_{\rho}, \mathcal{A M}_{g}(X)$ is the moduli space of such data. It is an Artin stack, fibered over the moduli space of quasi-stable curves [36]. We hope to use this refined moduli to do computations for higher genus mirror principle.

On the other hand, motivated by recent progresses in open string theory, we are also trying to develop open mirror principle. Open string theory predicts formulas for the counting of holomorphic discs with boundary inside a Lagrangian submanifold, more generally of the counting of the numbers of open Riemann surfaces with boundary in Lagrangian submanifold. Linearized moduli space for such data is being constructed which gives a new compactification of such moduli spaces.

\section{Hori-VAfa Formula}

In [17, Hori and Vafa generalize the world-sheet aspects of mirror symmetry to being the equivalence of $d=2, N=(2,2)$ supersymmetric field theories (i.e. without imposing the conformal invariance on the theory). This leads them to a much broader encompassing picture of mirror symmetry. Putting this in the frame work of abelian gauged linear sigma models (GLSM) of Witten enables them to link many $d=2$ field theories together. Generalization of this setting to nonabelian GLSM leads them to the following conjecture, when the physical path integrals are interpreted appropriately mathematically: 
Conjecture 4.1 (Hori-Vafa [17]). The hypergeometric series for a given homogeneous space (e.g. a Grassmannian manifold) can be reproduced from the hypergeometric series of simpler homogeneous spaces (e.g. product of projective spaces). Similarly for the twisted hypergeometric series that are related to the submanifolds in homogeneous spaces.

In other words, different homogeneous spaces (or some simple quotients of them) can give rise to generalized mirror pairs. A main object to be understood in the above conjecture is the fundamental hypergeometric series $H G[\mathbf{1}]^{X}(t)$ associated to the flag manifold $X$. Recall that in the computations of mirror principle, the existence of linearized moduli made easy the computations for toric manifolds.

An outline of how this series may be computed was given in [33] via an extended mirror principle diagram. To make clear the main ideas we will only focus on the case of Grassmannian manifolds in this article. The main problem for the computation is that there is no known good linearized moduli for Grassmannian or general flag manifolds. To overcome the difficulty we use the Grothendieck quot scheme to play the role of the linearized moduli. The method gives a complete proof of the Hori-Vafa formula in the Grassmannian case.

Let $e v: \overline{\mathcal{M}}_{0,1}(d, X) \rightarrow X$ be the evaluation map on the moduli space of stable maps with one marked point, and $c$ the first Chern class of the tangent line at the marked point. The fundamental hypergeometric series for mirror formula is given by the push-forward:

$$
e v_{*}\left[\frac{1}{\alpha(\alpha-c)}\right] \in H^{*}(X)
$$

or more precisely the generating series

$$
H G[1]^{X}(t)=e^{-t H / \alpha} \sum_{d=0}^{\infty} e v_{*}\left[\frac{1}{\alpha(\alpha-c)}\right] e^{d t} .
$$

Assume the linearized moduli exists. Then functorial localization formula applied to the collapsing map: $\varphi: M_{d} \rightarrow N_{d}$, immediately gives the expression as the denominator of the hypergeometric series.

Example. $X=\mathbf{P}^{n}$, then we have $\varphi_{*}(1)=1$, functorial localization immediately gives us

$$
e v_{*}\left[\frac{1}{\alpha(\alpha-c)}\right]=\frac{1}{\prod_{m=1}^{d}(x-m \alpha)^{n+1}}
$$

where the denominators of both sides are equivariant Euler classes of normal bundles of the fixed points. Here $x$ denotes the hyperplane class.

For $X=\operatorname{Gr}(k, n)$ or general flag manifolds, no explicit linearized moduli is known. Hori-Vafa conjectured a formula for $H G[1]^{X}(t)$ by which we can compute this series in terms of those of projective spaces which is the Hori-Vafa formula for Grassmannians:

Theorem 4.2. We have

$$
H G[1]^{\operatorname{Gr}(k, n)}(t)=\left.\frac{e^{(k-1) \pi \sqrt{-1} \sigma / \alpha}}{\prod_{i<j}\left(x_{i}-x_{j}\right)} \cdot \prod_{i<j}\left(\alpha \frac{\partial}{\partial x_{i}}-\alpha \frac{\partial}{\partial x_{j}}\right)\right|_{t_{i}=t+(k-1) \pi \sqrt{-1}} H G[1]^{\mathbf{P}}\left(t_{1}, \cdots, t_{k}\right)
$$

where $\mathbf{P}=\mathbf{P}^{n-1} \times \cdots \times \mathbf{P}^{n-1}$ is product of $k$ copies of the projective spaces, $\sigma$ is the generator of the divisor classes on $\operatorname{Gr}(k, n)$ and $x_{i}$ the hyperplane class of the $i$-th copy 
$\mathbf{P}^{n-1}$ :

$$
H G[1]^{\mathbf{P}}\left(t_{1}, \cdots, t_{k}\right)=\prod_{i=1}^{k} H G[1]^{\mathbf{P}^{\mathbf{n}-1}}\left(t_{i}\right) .
$$

Now we describe the ideas of the proof of the above formula. As mentioned above we use another smooth moduli space, the Grothendieck quot-scheme $Q_{d}$ to play the role of the linearized moduli, and apply the functorial localization formula. Here is the general set-up:

To start, note that the Plücker embedding $\tau: \operatorname{Gr}(k, n) \rightarrow \mathbf{P}^{N}$ induces an embedding of the nonlinear moduli $M_{d}$ of $\operatorname{Gr}(k, n)$ into that of $\mathbf{P}^{N}$. Composite of this map with the collapsing map gives us a map $\varphi: M_{d} \rightarrow W_{d}$ into the linearized moduli space $W_{d}$ of $\mathbf{P}^{N}$. On the other hand the Plücker embedding also induces a map $\psi: Q_{d} \rightarrow W_{d}$. We have the following three crucial lemmas proved in [30].

Lemma 4.3. The above two maps have the same image in $W_{d}: \operatorname{Im} \psi=\operatorname{Im} \varphi$. And all the maps are equivariant with respect to the induced circle action from $\mathbf{P}^{1}$.

Just as in the mirror principle computations, our next step is to analyze the fixed points of the circle action induced from $\mathbf{P}^{1}$. In particular we need the distinguished fixed point set to get the equivariant Euler class of its normal bundle. The distinguished fixed point set in $M_{d}$ is $\overline{\mathcal{M}}_{0,1}(d, \operatorname{Gr}(k, n))$ with equivariant Euler class of its normal bundle given by $\alpha(\alpha-c)$, and we know that $\varphi$ is restricted to $e v$.

Lemma 4.4. The distinguished fixed point set in $Q_{d}$ is a union: $\cup_{s} E_{0 s}$, where each $E_{0 s}$ is a fiber bundle over $\operatorname{Gr}(k, n)$ with fiber given by flag manifold.

It is a complicated work to determine the fixed point sets $E_{0 s}$ and the weights of the circle action on their normal bundles. The situation for flag manifold cases are much more involved. See [30] and [37] for details.

Now let $p$ denote the projection from $E_{0 s}$ onto $\operatorname{Gr}(k, n)$. Functorial localization formula, applied to $\varphi$ and $\psi$, gives us the following

Lemma 4.5. We have the equality on $\operatorname{Gr}(k, N)$ :

$$
e v_{*}\left[\frac{1}{\alpha(\alpha-c)}\right]=\sum_{s} p_{*}\left[\frac{1}{e_{T}\left(E_{0 s} / Q_{d}\right)}\right]
$$

where $e_{T}\left(E_{0 s} / Q_{d}\right)$ is the equivariant Euler class of the normal bundle of $E_{0 s}$ in $Q_{d}$.

Finally we compute $p_{*}\left[\frac{1}{e_{T}\left(E_{0 s} / Q_{d}\right)}\right]$. There are two different approaches, the first one is by direct computations in [30], and another one is by using the well-known Euler sequences for universal sheaves [4]. The second method has the advantage of being more explicit. Note that

$$
e_{T}\left(\left.T Q\right|_{E_{0 s}}-T E_{0 s}\right)=e_{T}\left(\left.T Q\right|_{E_{0 s}}\right) / e_{T}\left(T E_{0 s}\right) .
$$

Both $e_{T}\left(\left.T Q\right|_{E_{0 s}}\right)$ and $e_{T}\left(T E_{0 s}\right)$ can be written down explicitly in terms of the universal bundles on the flag bundle $E_{0 s}=F l\left(m_{1}, \cdots, m_{k}, S\right)$ over $\operatorname{Gr}(r, n)$. Here $S$ is the universal bundle on the Grassmannian.

The push-forward by $p$ from $F l\left(m_{1}, \cdots, m_{k}, S\right)$ to $\operatorname{Gr}(r, n)$ is done by an analogue of family localization formula of Atiyah-Bott, which is given by a sum over the Weyl groups along the fiber which labels the fixed point sets. 
In any case the final formula of degree $d$ is given by

$$
p_{*}\left[\frac{1}{e_{T}\left(E_{0 s} / Q_{d}\right)}\right]=(-1)^{(r-1) d} \sum_{\substack{\left(d_{1}, \ldots, d_{r}\right) \\ d_{1}+\ldots+d_{r}=d}} \frac{\prod_{1 \leq i<j \leq r}\left(x_{i}-x_{j}+\left(d_{i}-d_{j}\right) \alpha\right)}{\prod_{1 \leq i<j \leq r}\left(x_{i}-x_{j}\right) \prod_{i=1}^{r} \prod_{l=1}^{d_{i}}\left(x_{i}+l \alpha\right)^{n}} .
$$

Here $x_{1}, \ldots x_{r}$ are the Chern roots of $S^{*}$. As a corollary of our approach, we have the following:

Corollary 4.6. The Hori-Vafa conjecture holds for Grassmannian manifolds.

See [4] and [30] for the details. For the explicit forms of Hori-Vafa conjecture for general flag manifolds, see [37] and [5].

\section{The Mariño-Vafa Conjecture}

Our original motivation to study Hodge integrals was to find a general mirror formula for counting higher genus curves in Calabi-Yau manifolds. To generalize mirror principle to count the number of higher genus curves, we need to first compute Hodge integrals, i.e. the intersection numbers of the $\lambda$ classes and $\psi$ classes on the Deligne-Mumford moduli space of stable curves $\overline{\mathcal{M}}_{g, h}$. This moduli space is possibly the most famous and most interesting orbifold. It has been studied since Riemann, and by many Fields medalists for the past 50 years, from many different point of views. Still many interesting and challenging problems about the geometry and topology of these moduli spaces remain unsolved. String theory has motivated many fantastic conjectures about these moduli spaces including the famous Witten conjecture which is about the generating series of the integrals of the $\psi$-classes. We start with the introduction of some notations.

Recall that a point in $\overline{\mathcal{M}}_{g, h}$ consists of $\left(C, x_{1}, \ldots, x_{h}\right)$, a (nodal) curve $C$ of genus $g$, and $n$ distinguished smooth points on $C$. The Hodge bundle $\mathbb{E}$ is a rank $g$ vector bundle over $\overline{\mathcal{M}}_{g, h}$ whose fiber over $\left[\left(C, x_{1}, \ldots, x_{h}\right)\right]$ is $H^{0}\left(C, \omega_{C}\right)$, the complex vector space of holomorphic one forms on $C$. The $\lambda$ classes are the Chern Classes of $\mathbb{E}$,

$$
\lambda_{i}=c_{i}(\mathbb{E}) \in H^{2 i}\left(\overline{\mathcal{M}}_{g, h} ; \mathbb{Q}\right) .
$$

On the other hand, the cotangent line $T_{x_{i}}^{*} C$ of $C$ at the $i$-th marked point $x_{i}$ induces a line bundle $\mathbb{L}_{i}$ over $\overline{\mathcal{M}}_{g, h}$. The $\psi$ classes are the Chern classes:

$$
\psi_{i}=c_{1}\left(\mathbb{L}_{i}\right) \in H^{2}\left(\overline{\mathcal{M}}_{g, h} ; \mathbb{Q}\right) .
$$

Introduce the total Chern class

$$
\Lambda_{g}^{\vee}(u)=u^{g}-\lambda_{1} u^{g-1}+\cdots+(-1)^{g} \lambda_{g} .
$$

The Mariño-Vafa formula is about the generating series of the triple Hodge integrals

$$
\int_{\overline{\mathcal{M}}_{g, h}} \frac{\Lambda_{g}^{\vee}(1) \Lambda_{g}^{\vee}(\tau) \Lambda_{g}^{\vee}(-\tau-1)}{\prod_{i=1}^{h}\left(1-\mu_{i} \psi_{i}\right)},
$$

where $\tau$ is considered as a parameter here. Later we will see that it actually comes from the weight of the group action, and also from the framing of the knot. Taking Taylor expansions in $\tau$ or in $\mu_{i}$ one can obtain information on the integrals of the Hodge classes and the $\psi$-classes. The Marinõ-Vafa conjecture asserts that the generating series of such

triple Hodge integrals for all genera and any numbers of marked points can be expressed 
by a close formula which is a finite expression in terms of representations of symmetric groups, or Chern-Simons knot invariants.

We remark that the moduli spaces of stable curves have been the sources of many interests from mathematics to physics. Mumford has computed some low genus numbers. The Witten conjecture, proved by Kontsevich [22], is about the integrals of the $\psi$-classes.

Let us briefly recall the background of the conjecture. Mariño and Vafa [46] made this conjecture based on the large $N$ duality between Chern-Simons and string theory. It starts from the conifold transition. We consider the resolution of singularity of the conifold $X$ defined by

$$
\left\{\left(\begin{array}{cc}
x & y \\
z & w
\end{array}\right) \in \mathbf{C}^{4}: x w-y z=0\right\}
$$

in two different ways:

(1). Deformed conifold $T^{*} S^{3}$

$$
\left\{\left(\begin{array}{cc}
x & y \\
z & w
\end{array}\right) \in \mathbf{C}^{4}: x w-y z=\epsilon\right\}
$$

where $\epsilon$ a real positive number. This is a symplectic resolution of the singularity.

(2). Resolved conifold by blowing up the singularity, which gives the total space

$$
\tilde{X}=\mathcal{O}(-1) \oplus \mathcal{O}(-1) \rightarrow \mathbf{P}^{1}
$$

which is explicitly given by

$$
\begin{aligned}
\left\{\left(\left[Z_{0}, Z_{1}\right],\left(\begin{array}{cc}
x & y \\
z & w
\end{array}\right)\right)\right. & \left.\in \mathbf{P}^{1} \times \mathbf{C}^{4}: \begin{array}{l}
(x, y) \in\left[Z_{0}, Z_{1}\right] \\
(z, w) \in\left[Z_{0}, Z_{1}\right]
\end{array}\right\} \\
\tilde{X} & \subset \mathbf{P}^{1} \times \mathbf{C}^{4} \\
\downarrow & \\
X & \subset \quad \stackrel{\mathbf{C}^{4}}{ }
\end{aligned}
$$

The brief history of the development of the conjecture is as follows. In 1992 Witten first conjectured that the open topological string theory on the deformed conifold $T^{*} S^{3}$ is equivalent to the Chern-Simons gauge theory on $S^{3}$. Such idea was pursued further by Gopakumar and Vafa in 1998, and then by Ooguri and Vafa in 2000. Based on the above conifold transition, they conjectured that the open topological string theory on the deformed conifold $T^{*} S^{3}$ is equivalent to the closed topological string theory on the resolved conifold $\tilde{X}$. Ooguri-Vafa only considered the zero framing case. Later MarinõVafa generalized the idea to the non-zero framing case and discovered the beautiful formula for the generating series of the triple Hodge integrals. Recently Vafa and his collaborators systematically developed the theory, and for the past several years, they developed these duality ideas into the most effective tool to compute Gromov-Witten invariants on toric Calabi-Yau manifolds. The high point of their work is the theory of topological vertex. We refer to [46] and [1] for the details of the physical theory and the history of the development.

Starting with the proof of the Marinõ-Vafa conjecture [40], [41], we have developed a rather complete mathematical theory of topological vertex [28]. Many interesting consequences have been derived for the past year. Now let us see how the string theorists derived mathematical consequence from the above naive idea of string duality. First the Chern-Simons partition function has the form 


$$
\langle Z(U, V)\rangle=\exp (-F(\lambda, t, V))
$$

where $U$ is the holonomy of the $U(N)$ Chern-Simons gauge field around the knot $K \subset S^{3}$, and $V$ is an extra $U(M)$ matrix. The partition function $\langle Z(U, V)\rangle$ gives the Chern-Simons knot invariants of $K$.

String duality asserts that the function $F(\lambda, t, V)$ should give the generating series of the open Gromov-Witten invariants of $\left(\tilde{X}, L_{K}\right)$, where $L_{K}$ is a Lagrangian submanifold of the resolved conifold $\tilde{X}$ canonically associated to the knot $K$. More precisely by applying the t'Hooft large $N$ expansion, and the "canonical" identifications of parameters similar to mirror formula, which at level $k$ are given by

$$
\lambda=\frac{2 \pi}{k+N}, \quad t=\frac{2 \pi i N}{k+N},
$$

we get the partition function of the topological string theory on conifold $\tilde{X}$, and then on $\mathbf{P}^{1}$. which is just the generating series of the Gromov-Witten invariants. This change of variables is very striking from the point of view of mathematics.

The special case when $K$ is the unknot is already very interesting. In non-zero framing it gives the Mariño-Vafa conjectural formula. In this case $\langle Z(U, V)\rangle$ was first computed in the zero framing by Ooguri-Vafa and in any framing $\tau \in \mathbb{Z}$ by Mariño-Vafa [46]. Comparing with Katz-Liu's computations of $F(\lambda, t, V)$, Mariño-Vafa conjectured the striking formula about the generating series of the triple Hodge integrals for all genera and any number of marked points in terms of the Chern-Simons invariants, or equivalently in terms of the representations and combinatorics of symmetric groups. It is interesting to note that the framing in the Mariño-Vafa's computations corresponds to the choice of lifting of the circle action on the pair $\left(\tilde{X}, L_{\text {unknot }}\right)$ in Katz-Liu's localization computations. Both choices are parametrized by an integer $\tau$ which will be considered as a parameter in the triple Hodge integrals. Later we will take derivatives with respect to this parameter to get the cut-and-join equation.

It is natural to ask what mathematical consequence we can have for general duality, that is for general knots in general three manifolds, a first naive question is what kind of general Calabi-Yau manifolds will appear in the duality, in place of the conifold. Some special cases corresponding to the Seifert manifolds are known by gluing several copies of conifolds.

Now we give the precise statement of the Mariño-Vafa conjecture, which is an identity between the geometry of the moduli spaces of stable curves and Chern-Simons knot invariants, or the combinatorics of the representation theory of symmetric groups.

Let us first introduce the geometric side. For every partition $\mu=\left(\mu_{1} \geq \cdots \mu_{l(\mu)} \geq 0\right)$, we define the triple Hodge integral to be,

where the coefficient

$$
G_{g, \mu}(\tau)=A(\tau) \cdot \int_{\overline{\mathcal{M}}_{g, l(\mu)}} \frac{\Lambda_{g}^{\vee}(1) \Lambda_{g}^{\vee}(-\tau-1) \Lambda_{g}^{\vee}(\tau)}{\prod_{i=1}^{l(\mu)}\left(1-\mu_{i} \psi_{i}\right)},
$$

$$
A(\tau)=-\frac{\sqrt{-1}^{|\mu|+l(\mu)}}{|\operatorname{Aut}(\mu)|}[\tau(\tau+1)]^{l(\mu)-1} \prod_{i=1}^{l(\mu)} \frac{\prod_{a=1}^{\mu_{i}-1}\left(\mu_{i} \tau+a\right)}{\left(\mu_{i}-1\right) !} .
$$


The expressions, although very complicated, arise naturally from localization computations on the moduli spaces of relative stable maps into $\mathbf{P}^{1}$ with ramification type $\mu$ at $\infty$.

We now introduce the generating series

$$
G_{\mu}(\lambda ; \tau)=\sum_{g \geq 0} \lambda^{2 g-2+l(\mu)} G_{g, \mu}(\tau) .
$$

The special case when $g=0$ is given by

$$
\int_{\overline{\mathcal{M}}_{0, l(\mu)}} \frac{\Lambda_{0}^{\vee}(1) \Lambda_{0}^{\vee}(-\tau-1) \Lambda_{0}^{\vee}(\tau)}{\prod_{i=1}^{l(\mu)}\left(1-\mu_{i} \psi_{i}\right)}=\int_{\overline{\mathcal{M}}_{0, l(\mu)}} \frac{1}{\prod_{i=1}^{l(\mu)}\left(1-\mu_{i} \psi_{i}\right)}
$$

which is known to be equal to $|\mu|^{l(\mu)-3}$ for $l(\mu) \geq 3$, and we use this expression to extend the definition to the case $l(\mu)<3$.

Introduce formal variables $p=\left(p_{1}, p_{2}, \ldots, p_{n}, \ldots\right)$, and define

$$
p_{\mu}=p_{\mu_{1}} \cdots p_{\mu_{l(\mu)}}
$$

for any partition $\mu$. These $p_{\mu_{j}}$ correspond to $\operatorname{Tr} V^{\mu_{j}}$ in the notations of string theorists. The generating series for all genera and all possible marked points are defined to be

$$
G(\lambda ; \tau ; p)=\sum_{|\mu| \geq 1} G_{\mu}(\lambda ; \tau) p_{\mu}
$$

which encode complete information of the triple Hodge integrals we are interested in.

Next we introduce the representation theoretical side. Let $\chi_{\mu}$ denote the character of the irreducible representation of the symmetric group $S_{|\mu|}$, indexed by $\mu$ with $|\mu|=\sum_{j} \mu_{j}$. Let $C(\mu)$ denote the conjugacy class of $S_{|\mu|}$ indexed by $\mu$. Introduce

$$
\mathcal{W}_{\mu}(\lambda)=\prod_{1 \leq a<b \leq l(\mu)} \frac{\sin \left[\left(\mu_{a}-\mu_{b}+b-a\right) \lambda / 2\right]}{\sin [(b-a) \lambda / 2]} \cdot \frac{1}{\prod_{i=1}^{l(\nu)} \prod_{v=1}^{\mu_{i}} 2 \sin [(v-i+l(\mu)) \lambda / 2]}
$$

This has an interpretation in terms of quantum dimension in Chern-Simons knot theory.

We define the following generating series

$$
R(\lambda ; \tau ; p)=\sum_{n \geq 1} \frac{(-1)^{n-1}}{n} \sum_{\mu}\left[\sum_{\cup_{i=1}^{n} \mu^{i}=\mu} \prod_{i=1}^{n} \sum_{\left|\nu^{i}\right|=\left|\mu^{i}\right|} \frac{\chi_{\nu^{i}}\left(C\left(\mu^{i}\right)\right)}{z_{\mu^{i}}} e^{\sqrt{-1}\left(\tau+\frac{1}{2}\right) \kappa_{\nu^{i}} \lambda / 2} \mathcal{W}_{\nu^{i}}(\lambda)\right] p_{\mu}
$$

where $\mu^{i}$ are sub-partitions of $\mu, z_{\mu}=\prod_{j} \mu_{j} ! j^{\mu_{j}}$ and

$$
\kappa_{\mu}=|\mu|+\sum_{i}\left(\mu_{i}^{2}-2 i \mu_{i}\right)
$$

for a partition $\mu$ which is also standard for representation theory of symmetric groups. There is the relation $z_{\mu}=|\operatorname{Aut}(\mu)| \mu_{1} \cdots \mu_{l(\mu)}$.

Finally we can give the precise statement of the Mariño-Vafa conjecture:

Conjecture 5.1. We have the identity

$$
G(\lambda ; \tau ; p)=R(\lambda ; \tau ; p)
$$


Before discussing the proof of this conjecture, we first give several remarks.

\section{Remarks:}

1. This conjecture is a formula: $\mathrm{G}$ : Geometry $=\mathrm{R}$ : Representations, and the representations of symmetric groups are essentially combinatorics.

2. We note that each $G_{\mu}(\lambda, \tau)$ is given by a finite and closed expression in terms of the representations of symmetric groups:

$$
G_{\mu}(\lambda, \tau)=\sum_{n \geq 1} \frac{(-1)^{n-1}}{n} \sum_{\cup_{i=1}^{n} \mu^{i}=\mu} \prod_{i=1}^{n} \sum_{\left|\nu^{i}\right|=\left|\mu^{i}\right|} \frac{\chi_{\nu^{i}}\left(C\left(\mu^{i}\right)\right)}{z_{\mu^{i}}} e^{\sqrt{-1}\left(\tau+\frac{1}{2}\right) \kappa_{\nu^{i}} \lambda / 2} \mathcal{W}_{\nu^{i}}(\lambda)
$$

The generating series $G_{\mu}(\lambda, \tau)$ gives the values of the triple Hodge integrals for moduli spaces of curves of all genera with $l(\mu)$ marked points.

3. Note that an equivalent expression of this formula is the following non-connected generating series. In this situation we have a relatively simpler combinatorial expression:

$G(\lambda ; \tau ; p)^{\bullet}=\exp [G(\lambda ; \tau ; p)]=\sum_{|\mu| \geq 0}\left[\sum_{|\nu|=|\mu|} \frac{\chi_{\nu}(C(\mu))}{z_{\mu}} e^{\sqrt{-1}\left(\tau+\frac{1}{2}\right) \kappa_{\nu} \lambda / 2} \mathcal{W}_{\nu}(\lambda)\right] p_{\mu}$

According to Mariño and Vafa, this formula gives values for all Hodge integrals up to three Hodge classes. Lu proved that this is right if we combine with some previously known simple formulas about Hodge integrals.

4. By taking Taylor expansion in $\tau$ on both sides of the Mariño-Vafa formula, we have derived various Hodge integral identities in [42.

For examples, as easy consequences of the Mariño-Vafa formula and the cut-andjoin equation as satisfied by the above generating series, we have unified simple proofs of the $\lambda_{g}$ conjecture by comparing the coefficients in $\tau$ in the Taylor expansions of the two expressions,

$$
\int_{\overline{\mathcal{M}}_{g, n}} \psi_{1}^{k_{1}} \cdots \psi_{n}^{k_{n}} \lambda_{g}=\left(\begin{array}{c}
2 g+n-3 \\
k_{1}, \ldots, k_{n}
\end{array}\right) \frac{2^{2 g-1}-1}{2^{2 g-1}} \frac{\left|B_{2 g}\right|}{(2 g) !},
$$

for $k_{1}+\cdots+k_{n}=2 g-3+n$, and the following identities for Hodge integrals:

$$
\int_{\overline{\mathcal{M}}_{g}} \lambda_{g-1}^{3}=\int_{\overline{\mathcal{M}}_{g}} \lambda_{g-2} \lambda_{g-1} \lambda_{g}=\frac{1}{2(2 g-2) !} \frac{\left|B_{2 g-2}\right|}{2 g-2} \frac{\left|B_{2 g}\right|}{2 g},
$$

where $B_{2 g}$ are Bernoulli numbers. And

$$
\int_{\overline{\mathcal{M}}_{g, 1}} \frac{\lambda_{g-1}}{1-\psi_{1}}=b_{g} \sum_{i=1}^{2 g-1} \frac{1}{i}-\frac{1}{2} \sum_{\substack{g_{1}+g_{2}=g \\ g_{1}, g_{2}>0}} \frac{\left(2 g_{1}-1\right) !\left(2 g_{2}-1\right) !}{(2 g-1) !} b_{g_{1}} b_{g_{2}}
$$

where

$$
b_{g}= \begin{cases}1, & g=0 \\ \frac{2^{2 g-1}-1}{2^{2 g-1}} \frac{\left|B_{2 g}\right|}{(2 g) !}, & g>0 .\end{cases}
$$


Now let us look at how we proved this conjecture. This is joint work with Chiu-Chu Liu, Jian Zhou, see [39] and [40] for details.

The first proof of this formula is based on the Cut-and-Join equation which is a beautiful match of combinatorics and geometry. The details of the proof is given in [39] and [40]. First we look at the combinatorial side. Denote by $\left[s_{1}, \cdots, s_{k}\right]$ a $k$-cycle in the permutation group. We have the following two obvious operations:

1. Cut: a $k$-cycle is cut into an $i$-cycle and a $j$-cycle:

$$
[s, t] \cdot\left[s, s_{2}, \cdots, s_{i}, t, t_{2}, \cdots t_{j}\right]=\left[s, s_{2}, \cdots, s_{i}\right]\left[t, t_{2}, \cdots t_{j}\right] .
$$

2. Join: an $i$-cycle and a $j$-cycle are joined to an $(i+j)$-cycle:

$$
[s, t] \cdot\left[s, s_{2}, \cdots, s_{i}\right]\left[t, t_{2}, \cdots t_{j}\right]=\left[s, s_{2}, \cdots, s_{i}, t, t_{2}, \cdots t_{j}\right] .
$$

Such operations can be organized into differential equations which we call the cut-and-join equation.

Now we look at the geometry side. In the moduli spaces of stable maps, cut and join have the following geometric meaning:

1. Cut: one curve splits into two lower degree or lower genus curves.

2. Join: two curves are joined together to give a higher genus or higher degree curve.

The combinatorics and geometry of cut-and-join are reflected in the following two differential equations, which look like heat equation. It is easy to show that such equation is equivalent to a series of systems of linear ordinary differential equations by comparing the coefficients on $p_{\mu}$. These equations are proved either by easy and direct computations in combinatorics or by localizations on moduli spaces of relative stable maps in geometry. In combinatorics, the proof is given by direct computations and was explored in combinatorics in the mid 80s and later by Zhou [39] for this case. The differential operator on the right hand side corresponds to the cut-and-join operations which we also simply denote by $(C J)$.

\section{Lemma 5.2.}

$$
\frac{\partial R}{\partial \tau}=\frac{1}{2} \sqrt{-1} \lambda \sum_{i, j=1}^{\infty}\left((i+j) p_{i} p_{j} \frac{\partial R}{\partial p_{i+j}}+i j p_{i+j}\left(\frac{\partial R}{\partial p_{i}} \frac{\partial R}{\partial p_{j}}+\frac{\partial^{2} R}{\partial p_{i} \partial p_{j}}\right)\right) .
$$

On the geometry side the proof of such equation is given by localization on the moduli spaces of relative stable maps into the the projective line $\mathbf{P}^{1}$ with fixed ramifications at $\infty$ :

Lemma 5.3.

$$
\frac{\partial G}{\partial \tau}=\frac{1}{2} \sqrt{-1} \lambda \sum_{i, j=1}^{\infty}\left((i+j) p_{i} p_{j} \frac{\partial G}{\partial p_{i+j}}+i j p_{i+j}\left(\frac{\partial G}{\partial p_{i}} \frac{\partial G}{\partial p_{j}}+\frac{\partial^{2} G}{\partial p_{i} \partial p_{j}}\right)\right)
$$

The proof of the above equation is given in [39]. Together with the following 
Initial Value. : $\tau=0$,

$$
G(\lambda, 0, p)=\sum_{d=1}^{\infty} \frac{p_{d}}{2 d \sin \left(\frac{\lambda d}{2}\right)}=R(\lambda, 0, p) .
$$

which is precisely the Ooguri-Vafa formula and which has been proved previously for example in [52], we therefore obtain the equality which is the Mariño-Vafa conjecture by the uniqueness of the solution:

Theorem 5.4. We have the identity

$$
G(\lambda ; \tau ; p)=R(\lambda ; \tau ; p) .
$$

During the proof we note that the cut-and-join equation is encoded in the geometry of the moduli spaces of stable maps. In fact we later find the convolution formula of the following form, which is a relation for the disconnected version $G^{\bullet}=\exp G$,

$$
G_{\mu}^{\bullet}(\lambda, \tau)=\sum_{|\nu|=|\mu|} \Phi_{\mu, \nu}^{\bullet}(-\sqrt{-1} \tau \lambda) z_{\nu} K_{\nu}^{\bullet}(\lambda)
$$

where $\Phi_{\mu, \nu}^{\bullet}$ is the generating series of double Hurwitz numbers, and $z_{\nu}$ is the combinatorial constant appeared in the previous formulas. Equivalently this gives the explicit solution of the cut-and-join differential equation with initial value $K^{\bullet}(\lambda)$, which is the generating series of the integrals of certain Euler classes on the moduli spaces of relative stable maps to $\mathbf{P}^{1}$. See [38] for the derivation of this formula, and see [41] for the two partition analogue.

The Witten conjecture as proved by Kontsevich states that the generating series of the $\psi$-class integrals satisfy infinite number of differential equations. The remarkable feature of Mariño-Vafa formula is that it gives a finite close formula. In fact by taking limits in $\tau$ and $\mu_{i}$ 's one can obtain the Witten conjecture. A much simpler direct proof of the Witten conjecture was obtained recently by Kim and myself in [21]. We directly derived the recursion formula which implies both the Virasoro relations and the KdV equations.

The same argument as our proof of the conjecture gives a simple and geometric proof of the ELSV formula for Hurwitz numbers. It reduces to the fact that the push-forward of 1 is a constant in equivariant cohomology for a generically finite-to-one map. See [40] for more details.

We would like to briefly explain the technical details of the proof. The proof of the combinatorial cut-and-join formula is based on the Burnside formula and various simple results in symmetric functions. See [52], 29] and [40].

The proof of the geometric cut-and-join formula used the functorial localization formula in [31] and [32]. The virtual version of this formula was proved first applied to the virtual fundamental cycles in the computations of Gromov-Witten invariants in [32].

As remarked in previous sections the functorial localization formula is very effective and useful because we can use it to push computations on complicated moduli space to simpler moduli space. The moduli spaces used by mathematicians are usually the correct but complicated moduli spaces like the moduli spaces of stable maps, while the moduli spaces used by physicists are usually the simple but the wrong ones like the projective spaces. This functorial localization formula has been used successfully in the proof of the mirror formula [31], [32, the proof of the Hori-Vafa formula [30], and the easy proof of 
the ELSV formula [40]. Our first proof of the Mariño-Vafa formula also used this formula in a crucial way.

More precisely, let $\overline{\mathcal{M}}_{g}\left(\mathbf{P}^{1}, \mu\right)$ denote the moduli space of relative stable maps from a genus $g$ curve to $\mathbf{P}^{1}$ with fixed ramification type $\mu$ at $\infty$, where $\mu$ is a fixed partition. We apply the functorial localization formula to the divisor morphism from the relative stable map moduli space to the projective space,

$$
\operatorname{Br}: \overline{\mathcal{M}}_{g}\left(\mathbf{P}^{1}, \mu\right) \rightarrow \mathbf{P}^{r}
$$

where $r$ denotes the dimension of $\overline{\mathcal{M}}_{g}\left(\mathbf{P}^{1}, \mu\right)$. This is similar to the set-up of mirror principle, only with a different linearized moduli space, but in both cases the target spaces are projective spaces.

We found that the fixed points of the target $\mathbf{P}^{r}$ precisely labels the cut-and-join operations of the triple Hodge integrals. Functorial localization reduces the problem to the study of polynomials in the equivariant cohomology group of $\mathbf{P}^{r}$. We were able to squeeze out a system of linear equations which implies the cut-and-join equation. Actually we derived a stronger relation than the cut-and-join equation, while the cut-and-join equation we need for the Mariño-Vafa formula is only the very first of such kind of relations. See [40] for higher order cut-and-join equations.

As was known in infinite Lie algebra theory, the cut-and-join operator is closely related to and more fundamental than the Virasoro algebras in some sense.

Recently there have appeared two different approaches to the Mariño-Vafa formula. The first one is a direct derivation of the convolution formula which was discovered during our proof of the two partition analogue of the formula [41]. See [38] for the details of the derivation in this case. The second is by Okounkov-Pandhripande [47], they gave a different approach by using the ELSV formula as initial value, and as well as the $\lambda_{g}$ conjecture and other recursion relations from localization on the moduli spaces of stable maps to $\mathbf{P}^{1}$.

\section{Two Partition Formula}

The two partition analogue of the Mariño-Vafa formula naturally arises from the localization computations of the Gromov-Witten invariants of the open toric Calabi-Yau manifolds, as explained in [53].

To state the formula we let $\mu^{+}, \mu^{-}$be any two partitions. Introduce the Hodge integrals involving these two partitions:

$$
G_{\mu^{+}, \mu^{-}}(\lambda ; \tau)=B\left(\tau ; \mu^{+}, \mu^{-}\right) \cdot \sum_{g \geq 0} \lambda^{2 g-2} A_{g}\left(\tau ; \mu^{+}, \mu^{-}\right)
$$

where

$$
A_{g}\left(\tau ; \mu^{+}, \mu^{-}\right)=\int_{\overline{\mathcal{M}}_{g, l\left(\mu^{+}\right)+l\left(\mu^{-}\right)}} \frac{\Lambda_{g}^{\vee}(1) \Lambda_{g}^{\vee}(\tau) \Lambda_{g}^{\vee}(-\tau-1)}{\prod_{i=1}^{l\left(\mu^{+}\right)}\left(1-\mu_{i}^{+} \psi_{i}\right) \prod_{j=1}^{l\left(\mu^{-}\right)} \tau\left(\tau-\mu_{i}^{-} \psi_{j+l\left(\mu^{+}\right)}\right)}
$$

and

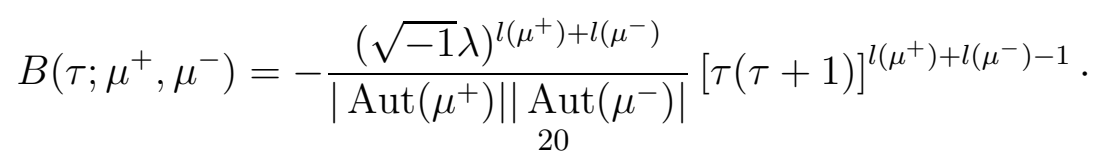




$$
\prod_{i=1}^{l\left(\mu^{+}\right)} \frac{\prod_{a=1}^{\mu_{i}^{+}-1}\left(\mu_{i}^{+} \tau+a\right)}{\left(\mu_{i}^{+}-1\right) !} \cdot \prod_{i=1}^{l\left(\mu^{-}\right)} \frac{\prod_{a=1}^{\mu_{i}^{-}-1}\left(\mu_{i}^{-} \frac{1}{\tau}+a\right)}{\left(\mu_{i}^{-}-1\right) !} .
$$

These complicated expressions naturally arise in open string theory, as well as in the localization computations of the Gromov-Witten invariants on open toric Calabi-Yau manifolds.

We introduce two generating series, first on the geometry side,

$$
G^{\bullet}\left(\lambda ; p^{+}, p^{-} ; \tau\right)=\exp \left(\sum_{\left(\mu^{+}, \mu^{-}\right) \in \mathcal{P}^{2}} G_{\mu^{+}, \mu^{-}}(\lambda, \tau) p_{\mu^{+}}^{+} p_{\mu^{-}}^{-}\right),
$$

where $\mathcal{P}^{2}$ denotes the set of pairs of partitions and $p_{\mu^{ \pm}}^{ \pm}$are two sets of formal variables associated to the two partitions as in the last section.

On the representation side, we introduce

$$
R^{\bullet}\left(\lambda ; p^{+}, p^{-} ; \tau\right)=\sum_{\left|\nu^{ \pm}\right|=\left|\mu^{ \pm}\right| \geq 0} \frac{\chi_{\nu^{+}}\left(C\left(\mu^{+}\right)\right)}{z_{\mu^{+}}} \frac{\chi_{\nu^{-}}\left(C\left(\mu^{-}\right)\right)}{z_{\mu^{-}}} \cdot e^{\sqrt{-1}\left(\kappa_{\nu^{+}} \tau+\kappa_{\nu^{-}} \tau^{-1}\right) \lambda / 2} \mathcal{W}_{\nu^{+}, \nu^{-}} p_{\mu^{+}}^{+} p_{\mu^{-}}^{-}
$$

Here

$$
\begin{gathered}
\mathcal{W}_{\mu, \nu}=q^{l(\nu) / 2} \mathcal{W}_{\mu} \cdot s_{\nu}\left(\mathcal{E}_{\mu}(t)\right) \\
=(-1)^{|\mu|+|\nu|} q^{\frac{\kappa_{\mu}+\kappa_{\nu}+|\mu|+|\nu|}{2}} \sum_{\rho} q^{-|\rho|} s_{\mu / \rho}(1, q, \ldots) s_{\nu / \rho}(1, q, \ldots)
\end{gathered}
$$

in terms of the skew Schur functions $s_{\mu}$ [45]. They appear naturally in the Chern-Simons invariant of the Hopf link.

Theorem 6.1. We have the identity:

$$
G^{\bullet}\left(\lambda ; p^{+}, p^{-} ; \tau\right)=R^{\bullet}\left(\lambda ; p^{+}, p^{-} ; \tau\right) .
$$

The idea of the proof is similar to that of the proof of the Mariño-Vafa formula. We prove that both sides of the above identity satisfy the same cut-and-join equation of the following type:

$$
\frac{\partial}{\partial \tau} H^{\bullet}=\frac{1}{2}(C J)^{+} H^{\bullet}-\frac{1}{2 \tau^{2}}(C J)^{-} H^{\bullet},
$$

where $(C J)^{ \pm}$denote the cut-and-join operator, the differential operator with respect to the two set of variables $p^{ \pm}$. We then prove that they have the same initial value at $\tau=-1$ :

$$
G^{\bullet}\left(\lambda ; p^{+}, p^{-} ;-1\right)=R^{\bullet}\left(\lambda ; p^{+}, p^{-} ;-1\right)
$$

which is again given by the Ooguri-Vafa formula [41, [53].

The cut-and-join equation can be written in a linear matrix form, and such equation follows from the convolution formula of the form

$$
K_{\mu^{+}, \mu^{-}}^{\bullet}(\lambda)=\sum_{\left|\nu^{ \pm}\right|=\mu^{ \pm}} G_{\mu^{+}, \mu^{-}}^{\bullet}(\lambda ; \tau) z_{\nu^{+}} \Phi_{\nu^{+}, \mu^{+}}^{\bullet}(-\sqrt{-1} \lambda \tau) z_{\nu^{-}} \Phi_{\nu^{-}, \mu^{-}}^{\bullet}\left(\frac{-\sqrt{-1}}{\tau} \lambda\right)
$$


where $\Phi^{\bullet}$ denotes the generating series of double Hurwitz numbers, and $K_{\mu^{+}, \mu^{-}}$is the generating series of certain integrals on the moduli spaces of relative stable maps. For more details see [41].

This convolution formula arises naturally from localization computations on the moduli spaces of relative stable maps to $\mathbf{P}^{1} \times \mathbf{P}^{1}$ with the point $(\infty, \infty)$ blown up. So it reflects the geometric structure of the moduli spaces. Such convolution type formula was actually discovered during our search for a proof of this formula, both on the geometric and the combinatorial side, see [41] for the detailed derivations of the convolution formulas in both geometry and combinatorics.

The proof of the combinatorial side of the convolution formula is again a direct computation. The proof of the geometric side for the convolution equation is to reorganize the generating series from localization contributions on the moduli spaces of relative stable maps into $\mathbf{P}^{1} \times \mathbf{P}^{1}$ with the point $(\infty, \infty)$ blown up, in terms of the double Hurwitz numbers. It involves careful analysis and computations.

\section{Theory of Topological Vertex}

When we worked on the Mariño-Vafa formula and its generalizations, we were simply trying to generalize the method and the formula to involve more partitions, but it turned out that in the three partition case, we naturally met the theory of topological vertex. Topological vertex was first introduced in string theory by Vafa et al in [1, it can be deduced from a three partition analogue of the Mariño-Vafa formula in a highly nontrivial way. From this we were able to give a rigorous mathematical foundation for the physical theory. Topological vertex is a high point of the theory of string duality as developed by Vafa and his group for the past several years, starting from Witten's conjectural duality between Chern-Simons and open string theory. It gives the most powerful and effective way to compute the Gromov-Witten invariants for all open toric Calabi-Yau manifolds. In physics it is rare to have two theories agree up to all orders, topological vertex theory gives a very significant example. In mathematics the theory of topological vertex already has many interesting applications. Here we only briefly sketch the rough idea for the three partition analogue of the Mariño-Vafa formula. For its relation to the theory of topological vertex, we refer the reader to [28] for the details.

Given any three partitions $\vec{\mu}=\left\{\mu^{1}, \mu^{2}, \mu^{3}\right\}$, the cut-and-join equation in this case, for both the geometry and representation sides, has the form:

$$
\frac{\partial}{\partial \tau} F^{\bullet}(\lambda ; \tau ; \mathbf{p})=(C J)^{1} F^{\bullet}(\lambda ; \tau ; \mathbf{p})+\frac{1}{\tau^{2}}(C J)^{2} F^{\bullet}(\lambda ; \tau ; \mathbf{p})+\frac{1}{(\tau+1)^{2}}(C J)^{3} F^{\bullet}(\lambda ; \tau ; \mathbf{p}) .
$$

The cut-and-join operators $(C J)^{1},(C J)^{2}$ and $(C J)^{3}$ are with respect to the three partitions. More precisely they correspond to the differential operators with respect to the three groups of infinite numbers of variables $\mathbf{p}=\left\{p^{1}, p^{2}, p^{3}\right\}$.

The initial value for this differential equation is taken at $\tau=1$, which is then reduced to the formulas of two partition case. The combinatorial, or the Chern-Simons invariant side is given by $\mathcal{W}_{\vec{\mu}}=\mathcal{W}_{\mu^{1}, \mu^{2}, \mu^{3}}$ which is a combination of the $\mathcal{W}_{\mu, \nu}$ as in the two partition case. See [28] for its explicit expression.

On the geometry side,

$$
G^{\bullet}(\lambda ; \tau ; \mathbf{p})=\exp _{22}(G(\lambda ; \tau ; \mathbf{p}))
$$


is the non-connected version of the generating series of the triple Hodge integral. More precisely,

$$
G(\lambda ; \tau ; \mathbf{p})=\sum_{\vec{\mu}}\left[\sum_{g=0}^{\infty} \lambda^{2 g-2+l(\vec{\mu})} G_{g, \vec{\mu}}(\tau)\right] p_{\mu^{1}}^{1} p_{\mu^{2}}^{2} p_{\mu_{3}}^{3}
$$

where $l(\vec{\mu})=l\left(\mu^{1}\right)+l\left(\mu^{2}\right)+l\left(\mu^{3}\right)$ and $G_{g, \vec{\mu}}(\tau)$ denotes the Hodge integrals of the following form,

$$
A(\tau) \int_{\overline{\mathcal{M}}_{g, l_{1}+l_{2}+l_{3}}} \frac{\Lambda_{g}^{\vee}(1) \Lambda_{g}^{\vee}(\tau) \Lambda_{g}^{\vee}(-\tau-1)}{\prod_{j=1}^{l_{1}}\left(1-\mu_{j}^{1} \psi_{j}\right) \prod_{j=1}^{l_{2}} \tau\left(\tau-\mu_{j}^{2} \psi_{l_{1}+j}\right)} \cdot \frac{(\tau(\tau+1))^{l_{1}+l_{2}+l_{3}-1}}{\prod_{j=1}^{l_{3}}(\tau+1)\left(\tau+1+\mu_{j}^{3} \psi_{l_{1}+l_{2}+j}\right)},
$$

where

$$
\begin{aligned}
& A(\tau)=\frac{-(\sqrt{-1} \lambda)^{l_{1}+l_{2}+l_{3}}}{\left|\operatorname{Aut}\left(\mu^{1}\right)\right|\left|\operatorname{Aut}\left(\mu^{2}\right)\right|\left|\operatorname{Aut}\left(\mu^{3}\right)\right|} \prod_{j=1}^{l_{1}} \frac{\prod_{a=1}^{\mu_{j}^{1}-1}\left(\tau \mu_{j}^{1}+a\right)}{\left(\mu_{j}^{1}-1\right) !} . \\
& \prod_{j=1}^{l_{2}} \frac{\prod_{a=1}^{\mu_{j}^{1}-1}\left((-1-1 / \tau) \mu_{j}^{2}+a\right)}{\left(\mu_{j}^{2}-1\right) !} \prod_{j=1}^{l_{3}} \frac{\prod_{a=1}^{\mu_{j}^{1}-1}\left(-\mu_{j}^{3} /(\tau+1)+a\right)}{\left(\mu_{j}^{3}-1\right) !}
\end{aligned}
$$

In the above expression, $l_{i}=l\left(\mu^{i}\right), i=1,2,3$. Despite of its complicated coefficients, these triple integrals naturally arise from localizations on the moduli spaces of relative stable maps into the blow-up of $\mathbf{P}^{1} \times \mathbf{P}^{1} \times \mathbf{P}^{1}$ along certain divisors. It also naturally appears in open string theory computations [1]. See [28] for more details.

One of our results in [28] states that $G^{\bullet}(\lambda ; \tau ; \mathbf{p})$ has a combinatorial expression $R^{\bullet}(\lambda ; \tau ; \mathbf{p})$ in terms of the Chern-Simons knot invariants $W_{\vec{\mu}}$, which is a closed combinatorial expression. More precisely it is given by

$$
R^{\bullet}(\lambda ; \tau ; \mathbf{p})=\sum_{\vec{\mu}}\left[\sum_{\left|\nu^{i}\right|=\left|\mu^{i}\right|} \prod_{i=1}^{3} \frac{\chi_{\nu^{i}}\left(\mu^{i}\right)}{z_{\mu^{i}}} q^{\frac{1}{2}\left(\sum_{i=1}^{3} \kappa_{\nu i} \frac{w_{i+1}}{w_{i}}\right)} W_{\vec{\nu}}(q)\right] p_{\mu^{1}}^{1} p_{\mu^{2}}^{2} p_{\mu^{3}}^{3} .
$$

Here $w_{4}=w_{1}$ and $w_{3}=-w_{1}-w_{2}$ and $\tau=\frac{w_{2}}{w_{1}}$. Due to the complicated combinatorics in the initial values, the combinatorial expression $W_{\vec{\mu}}$ we obtained is different from the expression $\mathcal{W}_{\vec{\mu}}$ obtained by Vafa et al. Actually our expression is even simpler than theirs in some sense. The expression we obtained is more convenient for mathematical applications such as the proof of the Gopakumar-Vafa conjecture for open toric CalabiYau manifolds, see [48].

Theorem 7.1. We have the equality:

$$
G^{\bullet}(\lambda ; \tau ; \boldsymbol{p})=R^{\bullet}(\lambda ; \tau ; \boldsymbol{p}) .
$$

The key point to prove the above theorem is still the proof of convolution formulas for both sides which imply the cut-and-join equation. The proof of the convolution formula for $G^{\bullet}(\lambda ; \tau ; \mathbf{p})$ is much more complicated than the one and two partition cases. See [28] for details.

The most useful property of topological vertex is its gluing property induced by the orthogonal relations of the characters of the symmetric group. This is very close to the 
situation of two dimensional gauge theory. In fact string theorists consider topological vertex as a kind of lattice theory on Calabi-Yau manifolds. By using the gluing formula we can easily obtain closed formulas for generating series of Gromov-Witten invariants of all genera and all degrees, open or closed, for all open toric Calabi-Yau manifolds, in terms of the Chern-Simons knot invariants. Such formulas are always given by finite sum of products of those Chern-Simons type invariants $\mathcal{W}_{\mu, \nu}$ 's. The magic of topological vertex is that, by simply looking at the moment map graph of the toric surfaces in the open toric Calabi-Yau, we can immediately write down the closed formula for the generating series for all genera and all degree Gromov-Witten invariants, or more precisely the Euler numbers of certain bundles on the moduli space of stable maps.

Here we only give one example to describe the topological vertex formula for the generating series of the all degree and all genera Gromov-Witten invariants for the open toric Calabi-Yau 3-folds. We write down the explicit close formula of the generating series of the Gromov-Witten invariants in this case.

Example: Consider the toric Calabi-Yau manifold which is $O(-3) \longrightarrow \mathbf{P}^{2}$. In this case the formula for the generating series of all degrees and all genera Gromov-Witten invariants is given by

$$
\exp \left(\sum_{g=0}^{\infty} \lambda^{2 g-2} F_{g}(t)\right)=\sum_{\nu_{1}, \nu_{2}, \nu_{3}} \mathcal{W}_{\nu_{1}, \nu_{2}} \mathcal{W}_{\nu_{2}, \nu_{3}} \mathcal{W}_{\nu_{3}, \nu_{1}}(-1)^{\sum_{j=1}^{3}\left|\nu_{j}\right|} q^{\frac{1}{2} \sum_{i=1}^{3} \kappa_{\nu_{i}}} e^{t\left(\sum_{j=1}^{3}\left|\nu_{j}\right|\right)}
$$

where $q=e^{\sqrt{-1} \lambda}$. The precise definition of $F_{g}(t)$ will be given in the next section.

For general open toric Calabi-Yau manifolds, the expressions are just similar. They are all given by finite and closed formulas, which are easily read out from the moment map graphs associated to the toric surfaces, with the topological vertex associated to each vertex of the graph.

In [1] Vafa and his group first developed the theory of topological vertex by using string duality between Chern-Simons and Calabi-Yau, which is a physical theory. In [28] we established the mathematical theory of the topological vertex, and derived various mathematical corollaries, including the relation of the Gromov-Witten invariants to the equivariant index theory as motivated by the Nekrasov conjecture in string duality [39]. During the development of the mathematical theory of topological vertex we also introduced formal Calabi-Yau manifolds, see [28] for details.

\section{Gopakumar-Vafa Conjecture and Indices of Elliptic Operators}

Let $N_{g, d}$ denote the so-called Gromov-Witten invariant of genus $g$ and degree $d$ of an open toric Calabi-Yau 3-fold. $N_{g, d}$ is defined to be the Euler number of the obstruction bundle on the moduli space of stable maps of degree $d \in H_{2}(S, \mathbb{Z})$ from genus $g$ curve into the surface base $S$. The open toric Calabi-Yau manifold associated to the toric surface $S$ is the total space of the canonical line bundle $K_{S}$ on $S$. More precisely

$$
N_{g, d}=\int_{\substack{\left.\overline{\mathcal{M}}_{g}(S, d)\right]^{v}\\}} e\left(V_{g, d}\right)
$$


with $V_{g, d}=R^{1} \pi_{*} u^{*} K_{S}$ a vector bundle on the moduli space induced by the canonical bundle $K_{S}$. Here $\pi: U \rightarrow \overline{\mathcal{M}}_{g}(S, d)$ denotes the universal curve and $u$ can be considered as the evaluation or universal map. Let us write

$$
F_{g}(t)=\sum_{d \geq 0} N_{g, d} e^{-d \cdot t} .
$$

The Gopakumar-Vafa conjecture is stated as follows:

Conjecture 8.1. There exists an expression:

$$
\sum_{g=0}^{\infty} \lambda^{2 g-2} F_{g}(t)=\sum_{k=1}^{\infty} \sum_{g, d \geq 0} n_{d}^{g} \frac{1}{d}\left(2 \sin \frac{d \lambda}{2}\right)^{2 g-2} e^{-k d \cdot t},
$$

such that $n_{d}^{g}$ are integers, called instanton numbers.

Motivated by the Nekrasov duality conjecture between the four dimensional gauge theory and string theory, we are able to interpret the above integers $n_{d}^{g}$ as equivariant indices of certain elliptic operators on the moduli spaces of anti-self-dual connections [39]:

Theorem 8.2. For certain interesting cases, these $n_{d}^{g}$ 's can be written as equivariant indices on the moduli spaces of anti-self-dual connections on $\mathbb{C}^{2}$.

For more precise statement, we refer the reader to [29]. The interesting cases include open toric Calabi-Yau manifolds when $S$ is Hirzebruch surface. The proof of this theorem is to compare fixed point formula expressions for equivariant indices of certain elliptic operators on the moduli spaces of anti-self-dual connections with the combinatorial expressions of the generating series of the Gromov-Witten invariants on the moduli spaces of stable maps. They both can be expressed in terms of Young diagrams of partitions. We find that they agree up to certain highly non-trivial "mirror transformation", a complicated variable change. This result is not only interesting for the index formula interpretation of the instanton numbers, but also for the fact that it gives the first complete examples that the Gopakumar-Vafa conjecture holds for all genera and all degrees.

Recently P. Peng [48] has given the proof of the Gopakumar-Vafa conjecture for all open toric Calabi-Yau 3-folds by using our Chern-Simons expressions from the topological vertex. His method is to explore the property of the Chern-Simons expression in great detail with some clever observation about the form of the combinatorial expressions. On the other hand, Kim in [20] has derived some remarkable recursion formulas for Hodge integrals of all genera and any number of marked points, involving one $\lambda$-classes. His method is to add marked points in the moduli spaces and then follow the localization argument we used to prove the Mariño-Vafa formula.

\section{Two Proofs of the ELSV Formula}

In this section we describe two proofs of the ELSV formula, one is by direct localization and cut-and-join equation following our proof of the Mariño-Vafa formula, another one is to derive it from the Mariño-Vafa formula through a scaling limit.

Given a partition $\mu$ of length $l(\mu)$, denote by $H_{g, \mu}$ the Hurwitz numbers of almost simple Hurwitz covers of $\mathbb{P}^{1}$ of ramification type $\mu$ by connected genus $g$ Riemann surfaces. The ELSV formula [10, 16] states:

$$
H_{g, \mu}=\underset{25}{2 g-2+|\mu|+l(\mu)) ! I_{g, \mu}}
$$


where

$$
I_{g, \mu}=\frac{1}{|\operatorname{Aut}(\mu)|} \prod_{i=1}^{l(\mu)} \frac{\mu_{i}^{\mu_{i}}}{\mu_{i} !} \int_{\overline{\mathcal{M}}_{g, l(\mu)}} \frac{\Lambda_{g}^{\vee}(1)}{\prod_{i=1}^{l(\mu)}\left(1-\mu_{i} \psi_{i}\right)} .
$$

Define generating functions

$$
\begin{aligned}
\Phi_{\mu}(\lambda) & =\sum_{g \geq 0} H_{g, \mu} \frac{\lambda^{2 g-2+|\mu|+l(\mu)}}{(2 g-2+|\mu|+l(\mu)) !}, \\
\Phi(\lambda ; p) & =\sum_{|\mu| \geq 1} \Phi_{\mu}(\lambda) p_{\mu}, \\
\Psi_{\mu}(\lambda) & =\sum_{g \geq 0} I_{g, \mu} \lambda^{2 g-2+|\mu|+l(\mu)}, \\
\Psi(\lambda ; p) & =\sum_{|\mu| \geq 1} \Psi_{\mu}(\lambda) p_{\mu} .
\end{aligned}
$$

In terms of generating functions, the ELSV formula reads

$$
\Psi(\lambda ; p)=\Phi(\lambda ; p) .
$$

It was known that $\Phi(\lambda ; p)$ satisfies the following cut-and-join equation:

$$
\frac{\partial \Theta}{\partial \lambda}=\frac{1}{2} \sum_{i, j \geq 1}\left(i j p_{i+j} \frac{\partial^{2} \Theta}{\partial p_{i} \partial p_{j}}+i j p_{i+j} \frac{\partial \Theta}{\partial p_{i}} \frac{\partial \Theta}{\partial p_{j}}+(i+j) p_{i} p_{j} \frac{\partial \Theta}{\partial p_{i+j}}\right) .
$$

Later this equation was reproved by sum formula of symplectic Gromov-Witten invariants 23 .

The calculations in Section 7 and Appendix A of [39] shows that

$$
\begin{gathered}
\tilde{H}_{g, \mu}=(2 g-2+|\mu|+l(\mu)) ! I_{g, \mu} \\
\tilde{H}_{g, \mu}=(2 g-3+|\mu|+l(\mu)) !\left(\sum_{\nu \in J(\mu)} I_{g, \nu}+\sum_{\nu \in C(\mu)} I_{2}(\nu) I_{g-1, \nu}\right. \\
\left.+\sum_{g_{1}+g_{2}=g} \sum_{\nu^{1} \cup \nu^{2} \in C(\mu)} I_{3}\left(\nu^{1}, \nu^{2}\right) I_{g_{1}, \nu_{1}} I_{g_{2}, \nu_{2}}\right)
\end{gathered}
$$

where

$$
\tilde{H}_{g, \mu}=\int_{\left[\overline{\mathcal{M}}_{g, 0}\left(\mathbf{P}^{1}, \mu\right)\right]^{\mathrm{vir}}} \operatorname{Br}^{*} H^{r}
$$

is some relative Gromov-Witten invariant of $\left(\mathbf{P}^{1}, \infty\right)$, and $C(\mu), J(\mu), I_{1}, I_{2}, I_{3}$ are defined as in [23]. So we have

$$
\begin{aligned}
& (2 g-2+|\mu|+l(\mu)) I_{g, \mu} \\
= & \sum_{\nu \in J(\mu)} I_{g, \nu}+\sum_{\nu \in C(\mu)} I_{2}(\nu) I_{g-1, \nu}+\sum_{g_{1}+g_{2}=g} \sum_{\nu^{1} \cup \nu^{2} \in C(\mu)} I_{3}\left(\nu^{1}, \nu^{2}\right) I_{g_{1}, \nu_{1}} I_{g_{2}, \nu_{2}},
\end{aligned}
$$

which is equivalent to the statement that the generating function $\Psi(\lambda ; p)$ of $I_{g, \mu}$ also satisfies the cut-and-join equation. 
Any solution $\Theta(\lambda ; p)$ to the cut-and-join equation (9) is uniquely determined by its initial value $\Theta(0 ; p)$, so it remains to show that $\Psi(0 ; p)=\Phi(0 ; p)$. Note that $2 g-2+|\mu|+$ $l(\mu)=0$ if and only if $g=0$ and $\mu=(1)$, so

$$
\Psi(0 ; p)=H_{0,(1)} p_{1}, \quad \Phi(0 ; p)=I_{0,(1)} p_{1} .
$$

It is easy to see that $H_{0,(1)}=I_{0,(1)}=1$, so

$$
\Psi(0 ; p)=\Phi(0 ; p) \text {. }
$$

One can see geometrically that the relative Gromov-Witten invariant $\tilde{H}_{g, \mu}$ is equal to the Hurwitz number $H_{g, \mu}$. This together with (9) gives a proof of the ELSV formula presented in [39, Section 7] in the spirit of [16]. Note that $\tilde{H}_{g, \mu}=H_{g, \mu}$ is not used in the proof described above.

On the other hand we can deduce the ELSV formula as the limit of the Mariño-Vafa formula. By the Burnside formula, one easily gets the following expression (see e.g. [41]):

$$
\begin{aligned}
\Phi(\lambda ; p) & =\log \left(\sum_{\mu}\left(\sum_{|\nu|=|\mu|} \frac{\chi_{\nu}(\mu)}{z_{\mu}} e^{\kappa_{\nu} \lambda / 2} \frac{\operatorname{dim} R_{\nu}}{|\nu| !}\right) p_{\mu} .\right) \\
& =\sum_{n \geq 1} \frac{(-1)^{n-1}}{n} \sum_{\mu} \sum_{\cup_{i=1}^{n} \mu_{i}=\mu} \prod_{i=1}^{n} \sum_{\left|\nu_{i}\right|=\left|\mu_{i}\right|} \frac{\chi_{\nu_{i}}\left(\mu_{i}\right)}{z_{\mu_{i}}} e^{\kappa_{\nu_{i}} \lambda / 2} \frac{\operatorname{dim} R_{\nu_{i}}}{\left|\nu_{i}\right| !} p_{\mu} .
\end{aligned}
$$

The ELSV formula reads

$$
\Psi(\lambda ; p)=\Phi(\lambda ; p)
$$

where the left hand side is a generating function of Hodge integrals $I_{g, \mu}$, and the right hand side is a generating function of representations of symmetric groups. So the ELSV formula and the Mariño-Vafa formula are of the same type.

Actually, the ELSV formula can be obtained by taking a particular limit of the MariñoVafa formula $G(\lambda ; \tau ; p)=R(\lambda ; \tau ; p)$. More precisely, it is straightforward to check that

$$
\begin{aligned}
& \lim _{\tau \rightarrow 0} G\left(\lambda \tau ; \frac{1}{\tau} ;(\lambda \tau) p_{1},(\lambda \tau)^{2} p_{2}, \cdots\right) \\
= & \sum_{|\mu| \neq 0} \sum_{g=0}^{\infty} \sqrt{-1}^{2 g-2+|\mu|+l(\mu)} I_{g, \mu} \lambda^{2 g-2+|\mu|+l(\mu)} p_{\mu} \\
= & \Psi(\sqrt{-1} \lambda ; p)
\end{aligned}
$$

and

$$
\begin{aligned}
& \lim _{\tau \rightarrow 0} R\left(\lambda \tau ; \frac{1}{\tau} ;(\lambda \tau) p_{1},(\lambda \tau)^{2} p_{2}, \cdots\right) \\
= & \log \left(\sum_{\mu}\left(\sum_{|\nu|=|\mu|} \frac{\chi_{\nu}(C(\mu))}{z_{\mu}} e^{\sqrt{-1} \kappa_{\nu} \lambda / 2} \lim _{t \rightarrow 0}\left(t^{|\nu|} V_{\nu}(t)\right)\right) p_{\mu}\right) \\
= & \log \left(\sum_{\mu}\left(\sum_{|\nu|=|\mu|} \frac{\chi_{\nu}(C(\mu))}{z_{\mu}} e^{\sqrt{-1} \kappa_{\nu} \lambda / 2} \frac{1}{\prod_{x \in \nu} h(x)}\right) p_{\mu}\right) \\
= & \Phi(\sqrt{-1} \lambda ; p)
\end{aligned}
$$


where we have used

$$
\frac{1}{\prod_{x \in \nu} h(x)}=\frac{\operatorname{dim} R_{\nu}}{|\nu| !} .
$$

In this limit, the cut-and-join equation of $G(\lambda ; \tau ; p)$ and $R(\lambda ; \tau ; p)$ reduces to the cutand-join equation of $\Psi(\lambda ; p)$ and $\Phi(\lambda ; p)$, respectively.

\section{A Localization Proof of the Witten Conjecture}

The Witten conjecture for moduli spaces states that the generating series $F$ of the integrals of the $\psi$ classes for all genera and any number of marked points satisfies the $\mathrm{KdV}$ equations and the Virasoro constraint. For example the Virasoro constraint states that $F$ satisfies

$$
L_{n} \cdot F=0, n \geq-1
$$

where $L_{n}$ denote certain Virasoro operators to be given later.

Witten conjecture was first proved by Kontsevich using combinatorial model of the moduli space and matrix model, with later approaches by Okounkov-Pandhripande using ELSV formula and combinatorics, by Mirzakhani using Weil-Petersson volumes on moduli spaces of bordered Riemann surfaces.

I will present a much simpler proof by using functorial localization and asymptotics. This was done jointly with Y.-S. Kim in [21. This is also motivated by methods in proving conjectures from string duality. It should have more applications.

The basic idea of our proof is to directly prove the following recursion formula which, as derived in physics by Dijkgraaf, Verlinde and Verlinde by using quantum field theory, implies the Virasoro and the KdV equation for the generating series $F$ of the integrals of the $\psi$ classes:

Theorem 10.1. We have identity

$$
\begin{gathered}
\left\langle\tilde{\sigma}_{n} \prod_{k \in S} \tilde{\sigma}_{k}\right\rangle_{g}=\sum_{k \in S}(2 k+1)\left\langle\tilde{\sigma}_{n+k-1} \prod_{l \neq k} \tilde{\sigma}_{l}\right\rangle_{g}+\frac{1}{2} \sum_{a+b=n-2}\left\langle\tilde{\sigma}_{a} \tilde{\sigma}_{b} \prod_{l \neq a, b} \tilde{\sigma}_{l}\right\rangle_{g-1} \\
+\frac{1}{2} \sum_{\substack{S=X \cup Y, a+b=n-2, g_{1}+g_{2}=g}}\left\langle\tilde{\sigma}_{a} \prod_{k \in X} \tilde{\sigma}_{k}\right\rangle_{g_{1}}\left\langle\tilde{\sigma}_{b} \prod_{l \in Y} \tilde{\sigma}_{l}\right\rangle_{g_{2}} .
\end{gathered}
$$

Here $\tilde{\sigma}_{n}=(2 n+1) ! ! \psi^{n}$ and

$$
\left\langle\prod_{j=1}^{n} \tilde{\sigma}_{k_{j}}\right\rangle_{g}=\int_{\overline{\mathcal{M}}_{g, n}} \prod_{j=1}^{n} \tilde{\sigma}_{k_{j}} .
$$

The notation $S=\left\{k_{1}, \cdots, k_{n}\right\}=X \cup Y$.

To prove the above recursion relation, similar to the proof of the Mariño-Vafa formula, we first apply the functorial localization to the natural branch map from moduli space of relative stable maps $\overline{\mathcal{M}}_{g}\left(\mathbf{P}^{1}, \mu\right)$ to projective space $\mathbf{P}^{r}$ where $r=2 g-2+|\mu|+l(\mu)$ is the dimension of the moduli.

As discussed in last section we easily get the cut-and-join equation for one Hodge integral

$$
I_{g, \mu}=\frac{1}{\mid \text { Aut } \mu \mid} \prod_{i=1}^{n} \frac{\mu_{i}^{\mu_{i}}}{\mu_{i} !} \int_{\overline{\mathcal{M}}_{g, n}} \frac{\Lambda_{g}^{\vee}(1)}{\prod\left(1-\mu_{i} \psi_{i}\right)} .
$$


The equation we get has the form as discussed in last section, it is trivial corollary of the fact that the push-forward of 1 in equivariant cohomology by a map between equal dimension manifolds is a constant:

$$
\begin{aligned}
& (2 g-2+|\mu|+l(\mu)) I_{g, \mu} \\
= & \sum_{\nu \in J(\mu)} I_{g, \nu}+\sum_{\nu \in C(\mu)} I_{2}(\nu) I_{g-1, \nu}+\sum_{g_{1}+g_{2}=g} \sum_{\nu^{1} \cup \nu^{2} \in C(\mu)} I_{3}\left(\nu^{1}, \nu^{2}\right) I_{g_{1}, \nu_{1}} I_{g_{2}, \nu_{2}} .
\end{aligned}
$$

Note that more general formulas of such type was first found and proved by Kim in [20].

Write $\mu_{i}=N x_{i}$. Let $N$ go to infinity and expand in $x_{i}$, we get:

$$
\begin{gathered}
\sum_{i=1}^{n}\left[\frac{\left(2 k_{i}+1\right) ! !}{2^{k_{i}+1} k_{i} !} x_{i}^{k_{i}} \prod_{j \neq i} \frac{x_{j}^{k_{j}-\frac{1}{2}}}{\sqrt{2 \pi}} \int_{\overline{\mathcal{M}}_{g, n}} \prod \psi_{j}^{k_{j}}-\sum_{j \neq i} \frac{\left(x_{i}+x_{j}\right)^{k_{i}+k_{j}-\frac{1}{2}}}{\sqrt{2 \pi}} \prod_{l \neq i, j} \frac{x_{l}^{k_{l}-\frac{1}{2}}}{\sqrt{2 \pi}} \int_{\overline{\mathcal{M}}_{g, n-1}} \psi^{k_{i}+k_{j}-1} \prod \psi_{l}^{k_{l}}\right. \\
-\frac{1}{2} \sum_{k+l=k_{i}-2} \frac{(2 k+1) ! !(2 l+1) ! !}{2^{k_{i}} k_{i} !} x_{i}^{k_{i}} \prod_{j \neq i} \frac{x_{j}^{k_{j}-\frac{1}{2}}}{\sqrt{2 \pi}}\left[\int_{\overline{\mathcal{M}}_{g-1, n+1}} \psi_{1}^{k} \psi_{2}^{l} \prod \psi_{j}^{k_{j}}\right. \\
\left.\left.+\sum_{\substack{g_{1}+g_{2}=g, \nu_{1} \cup \nu_{2}=\nu}} \int_{\overline{\mathcal{M}}_{g_{1}, n_{1}}} \psi_{1}^{k} \prod \psi_{j}^{k_{j}} \int_{\overline{\mathcal{M}}_{g_{2}, n_{2}}} \psi_{1}^{l} \prod \psi_{j}^{k_{j}}\right]\right]=0 .
\end{gathered}
$$

Performing Laplace transforms on the $x_{i}$ 's, we get the recursion formula in the above theorem which implies both the KdV equations and the Virasoro constraints. For example the Virasoro constraints states that the generating series

$$
\tau(\tilde{t})=\exp \sum_{g=0}^{\infty}\left\langle\exp \sum_{n} \tilde{t}_{n} \tilde{\sigma}_{n}\right\rangle_{g}
$$

satisfies the equations:

$$
L_{n} \cdot \tau=0, \quad(n \geq-1)
$$

where $L_{n}$ denote the Virasoro differential operators

$$
\begin{aligned}
L_{-1} & =-\frac{1}{2} \frac{\partial}{\partial \tilde{t}_{0}}+\sum_{k=1}^{\infty}\left(k+\frac{1}{2}\right) \tilde{t}_{k} \frac{\partial}{\partial \tilde{t}_{k-1}}+\frac{1}{4} \tilde{t}_{0}^{2} \\
L_{0} & =-\frac{1}{2} \frac{\partial}{\partial \tilde{t}_{1}}+\sum_{k=0}^{\infty}\left(k+\frac{1}{2}\right) \tilde{t}_{k} \frac{\partial}{\partial \tilde{t}_{k}}+\frac{1}{16} \\
L_{n} & =-\frac{1}{2} \frac{\partial}{\partial \tilde{t}_{n-1}}+\sum_{k=0}^{\infty}\left(k+\frac{1}{2}\right) \tilde{t}_{k} \frac{\partial}{\partial \tilde{t}_{k+n}}+\frac{1}{4} \sum_{i=1}^{n} \frac{\partial^{2}}{\partial \tilde{t}_{i-1} \partial \tilde{t}_{n-i}}
\end{aligned}
$$

We remark the same method can be used to derive very general recursion formulas in Hodge integrals and general Gromov-Witten invariants. We hope to report these results on a later occasion. 


\section{Final Remarks}

We strongly believe that there is a more interesting and grand duality picture between Chern-Simons invariants for three dimensional manifolds and the Gromov-Witten invariants for open toric Calabi-Yau manifolds. Our proofs of the Mariño-Vafa formula, and the setup of the mathematical foundation for topological vertex theory and the results of others we have discussed above all together have just opened a small window for a more splendid picture. We can expect more exciting conjectures from such duality to stimuate more developments in mathematics.

\section{REFERENCES}

[1] M. Aganagic, A. Klemm, M. Marino, C. Vafa, The topological vertex, preprint, hep-th/0305132.

[2] M. Aganagic, M. Marino, C. Vafa, All loop topological string amplitudes from Chern-Simons theory, preprint, hep-th/0206164.

[3] G. Bini, C. De Concini, M. Polito, C. Procesi On the work of Givental relative to mirror symmetry, math.AG/9805097.

[4] A. Bertram, I. Ciocan-Fontanine and B. Kim, Two proofs of a conjecture of Hori and Vafa, math.AG/0304403

[5] A. Bertram, I. Ciocan-Fontanine and B. Kim, Gromov-Witten Invariants for Abelian and Nonabelian Quotients, math.AG/0407254

[6] K. Behrend and B. Fantechi, The intrinsic normal cone, Invent. Math. 128 (1997), 45-88.

[7] P.Candelas, X.C. de la Ossa, P.S. Green, L. Parkes An exactly soluble superconformal theory from a mirror pair of Calabi-Yau manifolds, Phys. Lett. B 258 (1991), no. 1-2, 118-126.

[8] D.A. Cox, The functor of a smooth toric variety, Tôhoku Math. J. (2)47 (1995), 251-262.

[9] D. Cox, S. Katz, Mirror Symmetry and Algebraic Geometry, Mathematical Surveys and Monographs 68, AMS, Providence, RI, 1999.

[10] T. Ekedahl, S. Lando, M. Shapiro, A. Vainshtein, Hurwitz numbers and intersections on moduli spaces of curves. Invent. Math. 146 (2001), no. 2, 297-327.

[11] C. Faber and R. Pandharipande, Hodge integrals and Gromov-Witten theory, Invent. Math. 139(1) (2000), 173-199.

[12] - Hodge integrals, partition matrices, and the $\lambda_{g}$ conjecture, Ann. of Math. (2) 157 (2003), no. $1,97-124$.

[13] A. Givental, Equivariant Gromov-Witten invariants, Internat. Math. Res. Notices 1996, no. 13, 613663.

[14] I.P. Goulden, D.M. Jackson, Combinatorial enumeration, John Wiley \& Sons, 1983.

[15] I.P. Goulden, D.M. Jackson, A. Vainshtein, The number of ramified coverings of the sphere by the torus and surfaces of higher genera, Ann. of Comb. 4 (2000), 27-46.

[16] T. Graber, R. Vakil, Hodge integrals and Hurwitz numbers via virtual localization, Compositio Math. 135 (2003), no. 1, 25-36.

[17] H. Hori and C. Vafa, Mirror symmetry, hep-th/0002222

[18] A. Iqbal, All genus topological amplitudes and 5-brane webs as Feynman diagrams, preprint, hep-th/0207114

[19] S. Katz, C.-C. Liu, Enumerative geometry of stable maps with Lagrangian boundary conditions and multiple covers of the disc, Adv. Theor. Math. Phys. 5 (2001), 1-49.

[20] Y. Kim, Computing Hodge integrals with one lambda-class, preprint, math-ph/0501018.

[21] Y. Kim, K. Liu A simple proof of Witten conjecture through localization, preprint, math.AG/0508384

[22] M. Kontsevich, Intersection theory on the moduli space of curves and the matrix Airy function. Comm. Math. Phys. 147 (1992), no. 1, 1-23.

[23] A. M. Li, G. Zhao, Q. Zheng, The number of ramified coverings of a Riemann surface by Riemann surface, Comm. Math. Phys. 213 (2000), no. 3, 685-696.

[24] J. Li, Stable Morphisms to singular schemes and relative stable morphisms, J. Diff. Geom. 57 (2001), 509-578. 
[25] J. Li, Relative Gromov-Witten invariants and a degeneration formula of Gromov-Witten invariants, J. Diff. Geom. 60 (2002), 199-293.

[26] J. Li, Lecture notes on relative $G W$-invariants, preprint.

[27] J. Li and G. Tian, Virtual moduli cycles and Gromov-Witten invariants of algebraic varieties, J. Amer. Math. Soc. 11 (1998), 119-174.

[28] J. Li, C.-C. Liu, K. Liu, J. Zhou, A mathematical theory of the topological vertex , preprint, math.AG/0411247.

[29] J. Li, K. Liu, J. Zhou, Topological string partition functions as equivariant indices, preprint, math.AG/0412089.

[30] B. Lian, C.-H. Liu, K. Liu, S.-T. Yau, The $S^{1}$-fixed points in quot-schemes and mirror principle computations, Contemp. Math., 322, 2003, 165-194.

[31] B. Lian, K. Liu, S.-T. Yau, Mirror Principle I, Asian J. Math., 1, 1997, 729-763.

[32] B. Lian, K. Liu, and S.-T. Yau, Mirror principle, II, Asian J. Math. 3 (1999), 109-146;

[33] B. Lian, K. Liu, S.-T. Yau, Mirror Principle III, Asian J. Math., 3, 1999, 771-800.

[34] B. Lian, K. Liu, and S.-T. Yau, Mirror principle, IV, math.AG/0007104.

[35] - A survey of mirror principle, math.AG/0010064.

[36] C.-H. Liu, K. Liu, and S.-T. Yau, On A-twisted moduli stack for curves from Witten's gauged linear sigma models, math.AG/0212316.

[37] — , $S^{1}$-fixed-points in hyper-Quot-schemes and an exact mirror formula for flag manifolds from the extended mirror principle diagram, math.AG/0401367.

[38] C.-C. Liu, Formulae of one-partition and two-partition Hodge integrals, preprint, math.AG/0502430

[39] C.-C. Liu, K. Liu, J. Zhou, On a proof of a conjecture of Mariño-Vafa on Hodge Integrals, Math. Res. Letters 11, 259-272(2004).

[40] C.-C. Liu, K. Liu, J. Zhou, A proof of a conjecture of Mariño-Vafa on Hodge Integrals, J. Differential Geometry, 65(2003) 289-340.

[41] C.-C, Liu, K. Liu, J. Zhou, A formula of two-partition Hodge integrals, math.AG/0310272, J. of AMS.

[42] C.-C, Liu, K. Liu, J. Zhou, Mariño-Vafa formula and Hodge integral identities, math.AG/0308015, J. Algebraic Geometry.

[43] K. Liu, Mathematical results inspired by physics, Proc. ICM 2002, Vol. III, 457-466.

[44] K. Liu, Modular Invariance and Characteristic Numbers, Commun. Math. Phys. 174 (1995), 29-42.

[45] I.G. MacDonald, Symmetric functions and Hall polynomials, 2nd edition.Claredon Press, 1995.

[46] M. Mariño, C. Vafa, Framed knots at large N, Orbifolds in mathematics and physics (Madison, WI, 2001), 185-204, Contemp. Math., 310, Amer. Math. Soc., Providence, RI, 2002.

[47] A. Okounkov, R. Pandharipande, Hodge integrals and invariants of the unknots, preprint, math.AG/0307209

[48] P. Pan, A simple proof of Gopakumar-Vafa conjecture for local toric Calabi-Yau manifolds, preprint, math.AG/0410540.

[49] R. Pandhripande, Rational curves on hypersurfaces (after A. Givental), Astrisque 252 (1998), 307340.

[50] E. Witten, Quantum field theory and the Jones polynomial, Commun. Math. Phys. 121 (1989) 351399.

[51] E. Witten, Two-dimensional gravity and intersection theory on moduli space. Surveys in differential geometry (Cambridge, MA, 1990), 243-310, Lehigh Univ., Bethlehem, PA, 1991.

[52] J. Zhou, Hodge integrals, Hurwitz numbers, and symmetric groups, preprint, math.AG/0308024.

[53] J. Zhou, A conjecture on Hodge integrals, preprint.

[54] J. Zhou, Localizations on moduli spaces and free field realizations of Feynman rules, preprint.

Center of Mathematical Sciences, Zhejiang University, Hangzhou, China, and Department of Mathematics, University of California at Los Angeles, Los Angeles, CA 900951555, USA, EMAIL: LIU@MATH.UCLA.EDU, LIU@CMS.ZJU.EDU.CN 